\title{
Towards the higher point holographic momentum space amplitudes. Part II. Gravitons
}

\author{
Soner Albayrak ${ }^{a, b}$ and Savan Kharel ${ }^{a, c}$ \\ ${ }^{a}$ Department of Physics, Yale University, \\ New Haven, CT 06511, U.S.A. \\ ${ }^{b}$ Walter Burke Institute for Theoretical Physics, Caltech, \\ Pasadena, CA 91125, U.S.A. \\ ${ }^{c}$ Department of Physics, Williams College, \\ Williamstown, MA 01267, U.S.A. \\ E-mail: soner.albayrak@yale.edu, sk20@williams.edu
}

\begin{abstract}
In this follow up paper, we calculate higher point tree level graviton Witten diagrams in $\mathrm{AdS}_{4}$ via bulk perturbation theory. We show that by rearranging the bulk to bulk graviton propagators, the calculations effectively reduce to the computation of a scalar factor. Analogous to the amplitudes for vector boson interactions we computed in the previous paper, scalar factors for the graviton exchange diagrams also become relatively simple when written in momentum space. We explicitly calculate higher point correlators and discuss how this momentum space formalism makes flat space and collinear limits simpler.
\end{abstract}

Keywords: Scattering Amplitudes, AdS-CFT Correspondence, Conformal Field Theory, Space-Time Symmetries

ArXiv ePrint: 1908.01835 


\section{Contents}

1 Introduction 1

2 Preliminaries 3

2.1 Review: AdS momentum space perturbation theory 3

2.2 Stripping off the tensorial part of the propagator 4

2.3 Bulk point integrated expressions 6

3 Amplitudes $\quad 8$

3.1 A basic Witten diagram: three point function 8

$\begin{array}{ll}3.2 & \text { Four point function }\end{array}$

$\begin{array}{lll}3.3 & \text { Five point function } & 11\end{array}$

$4 \quad$ Flat space and collinear limits $\quad 12$

$\begin{array}{lll}5 & \text { Conclusion } & 15\end{array}$

$\begin{array}{ll}\text { A Scalar factor for five point diagram } & 16\end{array}$

\section{Introduction}

The AdS/CFT correspondence maps gravitational theories with their non-gravitational counterparts $[1,2]$. This correspondence has provided a concrete tool to tackle problems in theoretical physics from the nature of black holes to non-equilibrium phenomena in strongly coupled systems including condensed matter physics. ${ }^{1}$

In Minkowski space, the S-matrix provides the transition amplitude for a set of particles in an asymptotic in state at $t=-\infty$ that turns into a different set of out states at $t=+\infty .^{2}$ In an AdS with a time-like boundary such notions of in and out states are not very sensible. Instead, one can view the AdS as a box where particles can interact perpetually. However, one can also alter the boundary conditions at the time-like boundary thereby creating and annihilating particles. We know from AdS/CFT correspondence that the transition amplitudes between these type of states are equal to the correlation functions of the dual conformal field theory (CFT) [9]. Such amplitudes are among the most fundamental objects, and many important observables of the theories living in AdS are constructed through them. Because of their importance, they have garnered appreciable interest in the last decade. ${ }^{3}$

\footnotetext{
${ }^{1}$ For a more recent introduction to AdS/CFT, see [3]. For a complementary review on conformal field theories, see [4].

${ }^{2}$ The standard Haag-Ruelle construction of S-matrix [5-7] assumes a mass gap in the spectrum hence S-matrix is not rigorously defined for massless particles. For a recent short discussion with an improved construction, see [8].

${ }^{3}$ For instance, early work in this direction was pioneered in these papers: [10-14].
} 
The study of the scattering amplitudes of gauge theories and gravity has revealed remarkably simple structures in flat space and has led to menagerie of basis such as twistors and geometric formulations like the amplituhedron [15-19]. Additionally, in the last several years we have witnessed inspiring representations which have helped in showing similarities between scattering amplitudes in flat space and their AdS counterparts [9, 20-33]. One related insight has come out of the recent investigation of holographic momentum space which indicates an interesting connection between momentum space Witten diagrams and flat space scattering amplitudes. Indeed, one can obtain the S-matrix from the AdS correlation function with an elegant limit $k_{1}+k_{2}+\cdots k_{n} \mapsto 0$ where $k_{i}$ is the magnitude of the external momenta [34, 35]. A similar flat space limit also exist in de Sitter space [36, 37]. In fact, anti-de Sitter and de Sitter correlators are closely related and this intimacy provides a cosmological motivation for studying AdS amplitudes as well. ${ }^{4}$ In that context, we are interested in studying the late time spatial correlations that encode the primordial scattering processes.

While many exciting directions have been explored in the study of conformal structure in momentum space [34-37, 39-55], a lot of work still remains to be done. In our previous paper, we used momentum space formalism of [41] to compute the explicit expressions for several higher point vector correlators which take surprisingly simple forms [56]. Furthermore, it is shown in [38] that these calculation can be considerably simplified in a judiciously chosen basis. Specifically, different coefficients of the terms in the tensor structure of a vector amplitude are all related to a specific one among them, which means that the whole calculation reduces to only one integration. ${ }^{5}$ In this follow-up paper, we will discuss an analogous method to reduce the momentum space graviton calculations to computation of a scalar factor, and we will explicitly compute tree level higher point Witten diagrams with exchanged gravitons. Furthermore, we will discuss the flat space and collinear limits in our settings and provide explicit results. We think that our results could serve as data points from which further insights can be drawn. For instance, the inflationary cosmology has stimulated a great deal of excitement in the study of late time de Sitter correlators [57-60] and we believe that the analogous calculations of momentum space AdS amplitudes can assist in the study of the shape of non-Gaussianities [36, 37, 53, 61-76].

Here is the organization of the paper. In section 2, we briefly summarize momentum space perturbation theory of scalars and vector bosons, and present an overview of the gravitons. We also discuss how to strip off the tensor part of the graviton bulk to bulk propagator and how to effectively compute the remaining scalar factor for any tree level Witten diagram by introducing bulk point integrated expressions. In section 3, we use these ingredients and explicitly compute three, four and five point functions. In section 4 we obtain the expected flat space expressions, and further comment on how specific collinear limits can be simplified in our construction. Finally, we comment on many promising directions in the conclusion.

\footnotetext{
${ }^{4}$ Even though mathematical framework of AdS and dS are intuitively related, their connection can be obscure. For example, it is shown in a recent paper [38] that an algorithm for the conformally coupled scalars in dS can be directly used for gauge bosons in AdS, a relation which is not manifestly obvious.

${ }^{5}$ In [38], they also introduce an algebraic algorithm which bypasses the integration. Extension of this algorithm to gravitons is an open problem that we would like to return later; however, in this paper, we will stick to explicit integration.
} 


\section{Preliminaries}

\subsection{Review: AdS momentum space perturbation theory}

In this section we provide a brief review of momentum space bulk perturbation theory. We refer reader interested in scalars and vector bosons to [56] and the references therein; in this follow-up paper, we will present a very succinct overview with an emphasis on the gravitons.

In order to write a momentum space amplitude for a tree level Witten diagram, one needs to take the product of all relevant bulk to bulk and bulk to boundary propagators with the vertex factors, followed by an integration along the bulk radial direction. ${ }^{6}$ In the case of gluons in the axial gauge, the relevant ingredients for $\mathrm{AdS}_{d+1}$ are

$$
\begin{aligned}
A_{i}^{a}(\boldsymbol{k}, z) & =\boldsymbol{\epsilon}_{i}^{a} \sqrt{\frac{2}{\pi}}(k z)^{\frac{d-2}{2}} K_{\frac{d-2}{2}}(k z), \\
\mathcal{G}_{i j}\left(\boldsymbol{k} ; z, z^{\prime}\right) & =\left(z z^{\prime}\right)^{\frac{d-2}{2}} \int_{0}^{\infty} d \omega J_{\frac{d-2}{2}}(\omega z) \frac{\omega H_{i j}^{(\omega, \boldsymbol{k})}}{\left(\boldsymbol{k}^{2}+\omega^{2}-i \epsilon\right)} J_{\frac{d-2}{2}}\left(\omega z^{\prime}\right), \\
H_{i j}^{(\omega, \boldsymbol{k})} & :=-i\left(\eta_{i j}+\frac{\boldsymbol{k}_{i} \boldsymbol{k}_{j}}{\omega^{2}}\right) \\
V_{i j k}\left(\boldsymbol{k}_{1}, \boldsymbol{k}_{2}, \boldsymbol{k}_{3}\right) & :=\frac{i}{\sqrt{2}}\left(\eta_{i j}\left(\boldsymbol{k}_{1}-\boldsymbol{k}_{2}\right)_{k}+\eta_{j k}\left(\boldsymbol{k}_{2}-\boldsymbol{k}_{3}\right)_{i}+\eta_{k i}\left(\boldsymbol{k}_{3}-\boldsymbol{k}_{1}\right)_{j}\right), \\
V_{c}^{i j k l} & :=i \eta^{i k} \eta^{j l}-\frac{i}{2}\left(\eta^{i j} \eta^{k l}+\eta^{i l} \eta^{j k}\right) .
\end{aligned}
$$

for the bulk to boundary propagator $A_{i}^{a}$, the bulk to bulk propagator $\mathcal{G}_{i j}{ }^{7}$ and the vertex factors $V$ 's. In the equations above, $K_{\nu}(x)$ is the modified Bessel function of the second kind, $\eta_{i j}=\eta^{i j}$ is the boundary metric, $\boldsymbol{\epsilon}_{i}^{a}$ is the transverse polarization tensor $\left(\boldsymbol{\epsilon}_{i}^{a} \boldsymbol{k}^{i}=0\right)$, and $k$ is the norm of the external momenta, i.e. $k=\sqrt{\left|\boldsymbol{k}^{2}\right|}$. In this paper, we will stick to spacelike momenta, i.e. $\boldsymbol{k}^{2}=k^{2}$, as was done in [56]: one can analytically continue to timelike momenta, however the bulk to boundary propagators need to be modified accordingly [41].

As an example, the color-ordered expression for the $s$-channel four point Witten diagram is written as

$$
\begin{aligned}
& \mathcal{M}_{4 s}=\int_{0}^{\infty} \frac{d z}{z^{d+1}} \frac{d z^{\prime}}{z^{\prime d+1}} A_{i}\left(\boldsymbol{k}_{1}, z\right) A_{j}\left(\boldsymbol{k}_{2}, z\right) V^{i j k}\left(\boldsymbol{k}_{1}, \boldsymbol{k}_{2},-\boldsymbol{k}_{1}-\boldsymbol{k}_{2}\right) \mathcal{G}_{k l}\left(\boldsymbol{k}_{1}+\boldsymbol{k}_{2} ; z, z^{\prime}\right) \\
& \times z^{4} z^{\prime 4} V^{l m n}\left(\boldsymbol{k}_{1}, \boldsymbol{k}_{2}, \boldsymbol{k}_{1}+\boldsymbol{k}_{2}\right) A_{m}\left(\boldsymbol{k}_{3}, z^{\prime}\right) A_{n}\left(\boldsymbol{k}_{4}, z^{\prime}\right) .
\end{aligned}
$$

An explicit expression for $d=3$ for the above expression can be found in [56].

In the expression above, we included the factor $z^{4} z^{\prime 4}$. This was given as part of the overall prescription in [56] without a detailed explanation. It basically follows from the

\footnotetext{
${ }^{6}$ We work with the coordinates $\left\{z, k_{i}\right\}$ where $k_{i}$ are the Fourier transform of $x_{i}$ for the Poincaré patch $d s^{2}=z^{-2}\left(d z^{2}+\eta_{i j} d x^{i} d x^{j}\right)$. Therefore what we call momentum space amplitude is the amplitude in the coordinates $\left\{k_{i}\right\}$, which is obtained after the dependence on the bulk radius direction $z$ is integrated.

${ }^{7}$ The integration range in the bulk-to-bulk propagator follows from a Bessel function identity used in its derivation, i.e. $\int_{0}^{\infty} J_{\nu}(a t) J_{\nu}(b t) t d t=a^{-1} \delta(a-b)$.
} 
fact that the relevant inverse metric is $g^{i j}=z^{2} \eta^{i j}$ and both in three point and contact vertices we need two inverse metrics. So, to ease the notation in the vector calculations, we effectively took $g_{i j}=g^{i j}=\eta_{i j}=\eta^{i j}$ and inserted necessary $z$ factors at the end. ${ }^{8}$ In this paper, we present the tree point vertex factor and the propagators in contravariant and covariant forms respectively, so one does not need to worry about any additional $z$-factors.

The analogous set of ingredients to eq. (2.1) for gravitons in axial gauge is given by [41]:

$$
\begin{aligned}
h_{i j}(\boldsymbol{k}, z)= & \epsilon_{i j} \sqrt{\frac{2}{\pi}} z^{-2}(k z)^{\frac{d}{2}} K_{\frac{d}{2}}(k z) \\
\mathcal{G}_{a b, c d}\left(\boldsymbol{k} ; z, z^{\prime}\right)= & \frac{i\left(z z^{\prime}\right)^{\frac{d}{2}-2}}{2} \int_{0}^{\infty} d \omega J_{\frac{d}{2}}(\omega z) J_{\frac{d}{2}}\left(\omega z^{\prime}\right) \\
& \times \frac{\omega\left(H_{a c}^{(\omega, \boldsymbol{k})} H_{b d}^{(\omega, \boldsymbol{k})}+H_{a d}^{(\omega, \boldsymbol{k})} H_{b c}^{(\omega, \boldsymbol{k})}-\frac{2}{d-1} H_{a b}^{(\omega, \boldsymbol{k})} H_{c d}^{(\omega, \boldsymbol{k})}\right)}{k^{2}+\omega^{2}-i \epsilon} \\
\mathcal{V}_{\boldsymbol{k}_{1}, \boldsymbol{k}_{2}, \boldsymbol{k}_{3}}^{i j k l m n}:= & z^{8} V_{\boldsymbol{k}_{1}, \boldsymbol{k}_{2}, \boldsymbol{k}_{3}}^{i j k l m n}, \\
V_{\boldsymbol{k}_{1}, \boldsymbol{k}_{2}, \boldsymbol{k}_{3}}^{i j k l m n}:= & \left(\frac{\left(\boldsymbol{k}_{2}\right)^{i}\left(\boldsymbol{k}_{3}\right)^{j} \eta^{k m} \eta^{l n}}{4}-\frac{\left(\boldsymbol{k}_{2}\right)^{i}\left(\boldsymbol{k}_{3}\right)^{k} \eta^{j m} \eta^{l n}}{2}\right)+\text { permutations }
\end{aligned}
$$

where $\epsilon_{i j}$ is the symmetric traceless transverse polarization tensor, e.g. $\epsilon_{i j} \boldsymbol{k}^{i}=\epsilon_{i j} \eta^{i j}=0$ and $\epsilon_{i j}=\epsilon_{j i}$. We defined $V_{\boldsymbol{k}_{1}, \boldsymbol{k}_{2}, \boldsymbol{k}_{3}}^{i j k l m n}$ for convenience while $\mathcal{V}_{\boldsymbol{k}_{1}, \boldsymbol{k}_{2}, \boldsymbol{k}_{3}}^{i j k l m n}$ is the appropriate three point vertex factor.

The permutations in the vertex are generated by the permutation group element $\left(\boldsymbol{k}_{1} \boldsymbol{k}_{2} \boldsymbol{k}_{3}\right)(i k m)(j l n)$ in cycle notation. ${ }^{9}$ This is analogous to the vertex factor for the vector boson in eq. (2.1d) where the second and third terms can be obtained from the first one by the permutation $\left(\boldsymbol{k}_{1} \boldsymbol{k}_{2} \boldsymbol{k}_{3}\right)(i j k)$.

\subsection{Stripping off the tensorial part of the propagator}

Our goal in this part is to provide a prescription to simplify the propagator. The form of the graviton propagator as given in eq. (2.3b) shows that there are three different radial integrations we need to consider due to $\omega$ dependence of $H_{a c}^{(\omega, \boldsymbol{k})} H_{b d}^{(\omega, \boldsymbol{k})}$; however, it is not immediately clear if these integrations are related. In [38], the authors addressed a similar problem for the gluon propagator. By rewriting the gluon propagator as

$\mathcal{G}_{i j}\left(\boldsymbol{k} ; z, z^{\prime}\right)=\left(\eta_{i j}-\frac{\boldsymbol{k}_{i} \boldsymbol{k}_{j}}{k^{2}}\right) \int \omega d \omega \frac{\sqrt{z z^{\prime}} J_{\frac{1}{2}}(\omega z) J_{\frac{1}{2}}\left(\omega z^{\prime}\right)}{i\left(k^{2}+\omega^{2}-i \epsilon\right)}+\frac{\boldsymbol{k}_{i} \boldsymbol{k}_{j}}{k^{2}} \int d \omega \frac{k^{2}+\omega^{2}}{\omega} \frac{\sqrt{z z^{\prime}} J_{\frac{1}{2}}(\omega z) J_{\frac{1}{2}}\left(\omega z^{\prime}\right)}{i\left(k^{2}+\omega^{2}-i \epsilon\right)}$,

they introduced a decomposition of it; diagrammatically,

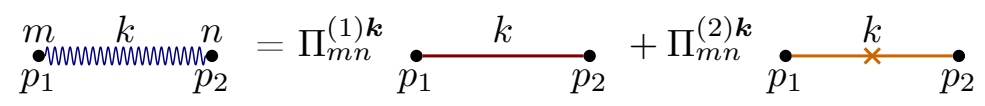

${ }^{8}$ For a similar discussion, see section 6.2 .2 of [41].

${ }^{9}$ See section 3.2.1. of [35] for the full contracted expression. 
for

$$
\Pi_{m n}^{(1) \boldsymbol{k}} \equiv-i\left(\eta_{m n}-\frac{\boldsymbol{k}_{m} \boldsymbol{k}_{n}}{k^{2}}\right), \quad \Pi_{m n}^{(2) \boldsymbol{k}} \equiv-i \frac{\boldsymbol{k}_{m} \boldsymbol{k}_{n}}{k^{2}}
$$

and

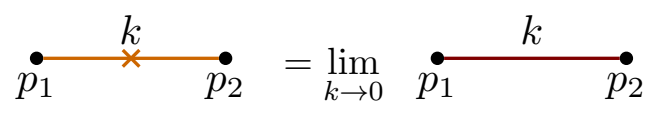

where

$$
\stackrel{\bullet}{p_{1}} \quad \quad \quad \quad \stackrel{p}{2}_{2}:=\int \omega d \omega \frac{\sqrt{z z^{\prime}} J_{\frac{1}{2}}(\omega z) J_{\frac{1}{2}}\left(\omega z^{\prime}\right)}{k^{2}+\omega^{2}-i \epsilon} .
$$

Using this decomposition, instead of performing $2^{n}$ integrations where $n$ is number of bulk to bulk propagator, one can only proceed with one explicit integration and obtain the rest via eq. (2.7).

We are actually abusing the notation in the equations above as these lines stand for bulk-point integrated diagrams in [38]. In the case of gluons, the additive property of the norm of momenta of the bulk to boundary propagators at the vertices enables performing computations at the level of truncated diagrams, hence the computations remain completely agnostic to what is attached from the boundary. This is no longer true for gravitons; thus, instead of working with the truncated graphs, we will work with the full Witten diagrams. Nonetheless, reducing the number of integrations to one by finding out an analogous relation for the graviton propagator will prove quite useful as we will see below.

To realize this goal, we first note the identity

$$
\begin{aligned}
\left(H_{a c}^{(\omega, \boldsymbol{k})} H_{b d}^{(\omega, \boldsymbol{k})}+H_{a d}^{(\omega, \boldsymbol{k})}\right. & \left.H_{b c}^{(\omega, \boldsymbol{k})}-\frac{2}{d-1} H_{a b}^{(\omega, \boldsymbol{k})} H_{c d}^{(\omega, \boldsymbol{k})}\right)=\mathcal{P}_{a b, c d}^{(1,1) \boldsymbol{k}} \\
& +\frac{k^{2}+\omega^{2}}{\omega^{2}}\left(\mathcal{P}_{a b, c d}^{(1,2) \boldsymbol{k}}+\mathcal{P}_{a b, c d}^{(2,1) \boldsymbol{k}}\right)+\left(\frac{k^{2}+\omega^{2}}{\omega^{2}}\right)^{2} \mathcal{P}_{a b, c d}^{(2,2) \boldsymbol{k}},
\end{aligned}
$$

where we define

$$
\mathcal{P}_{a b, c d}^{(m, n) \boldsymbol{k}} \equiv \Pi_{a c}^{(m) \boldsymbol{k}} \Pi_{b d}^{(n) \boldsymbol{k}}+\Pi_{a d}^{(m) \boldsymbol{k}} \Pi_{b c}^{(n) \boldsymbol{k}}-\frac{2}{d-1} \Pi_{a b}^{(m) \boldsymbol{k}} \Pi_{c d}^{(n) \boldsymbol{k}} .
$$

This enables us to rewrite the graviton propagator in eq. $(2.3 \mathrm{~b})$ as

$$
\begin{aligned}
\mathcal{G}_{a b, c d}\left(\boldsymbol{k} ; z, z^{\prime}\right)= & \frac{i \mathcal{P}_{a b, c d}^{(1,1) \boldsymbol{k}}}{2} \int \omega d \omega \frac{\left(z z^{\prime}\right)^{\frac{d}{2}-2} J_{\nu}(\omega z) J_{\nu}\left(\omega z^{\prime}\right)}{k^{2}+\omega^{2}-i \epsilon} \\
& +\frac{i\left(\mathcal{P}_{a b, c d}^{(1,2) \boldsymbol{k}}+\mathcal{P}_{a b, c d}^{(2,1) \boldsymbol{k}}+\mathcal{P}_{a b, c d}^{(2,2) \boldsymbol{k}}\right)}{2} \int \omega d \omega \frac{\left(z z^{\prime}\right)^{\frac{d}{2}-2} J_{\nu}(\omega z) J_{\nu}\left(\omega z^{\prime}\right)}{\omega^{2}} \\
& +\frac{i \mathcal{P}_{a b, c d}^{(2,2) \boldsymbol{k}}}{2} k^{2} \int \omega d \omega \frac{\left(z z^{\prime}\right)^{\frac{d}{2}-2} J_{\nu}(\omega z) J_{\nu}\left(\omega z^{\prime}\right)}{\omega^{4}}
\end{aligned}
$$

hence we obtain the desired form

$$
\mathcal{G}_{a b, c d}\left(\boldsymbol{k} ; z, z^{\prime}\right)=\mathcal{D}_{a b, c d}^{k} \int_{0}^{\infty} \omega d \omega \frac{\left(z z^{\prime}\right)^{\frac{d}{2}-2} J_{\nu}(\omega z) J_{\nu}\left(\omega z^{\prime}\right)}{k^{2}+\omega^{2}-i \epsilon},
$$


where we define the differential operator

$$
\mathcal{D}_{a b, c d}^{\boldsymbol{k}}:=\frac{i}{2}\left[\mathcal{P}_{a b, c d}^{(0) \boldsymbol{k}}+\mathcal{P}_{a b, c d}^{(1) \boldsymbol{k}} \lim _{k \rightarrow 0}-\mathcal{P}_{a b, c d}^{(2) \boldsymbol{k}} k^{2} \lim _{k \rightarrow 0} \partial_{k^{2}}\right]
$$

for

$$
\mathcal{P}_{a b, c d}^{(0) \boldsymbol{k}} \equiv \mathcal{P}_{a b, c d}^{(1,1) \boldsymbol{k}}, \quad \mathcal{P}_{a b, c d}^{(1) \boldsymbol{k}} \equiv \mathcal{P}_{a b, c d}^{(1,2) \boldsymbol{k}}+\mathcal{P}_{a b, c d}^{(2,1) \boldsymbol{k}}+\mathcal{P}_{a b, c d}^{(2,2) \boldsymbol{k}}, \quad \mathcal{P}_{a b, c d}^{(2) \boldsymbol{k}} \equiv \mathcal{P}_{a b, c d}^{(2,2) \boldsymbol{k}} .
$$

The nice thing about the operator $\mathcal{D}^{k}$ is that it commutes with the rest of the calculation, e.g. bulk point integration, so we can apply it at the very end. Furthermore, $\mathcal{D}^{k}$ for different propagators commute as well. Thus for a Witten diagram with $n$ bulk to bulk propagators we schematically have

$$
\mathcal{A}_{\mathrm{Witten}}=\left(\epsilon_{i}, V_{i}\right)^{a_{11} a_{12} \ldots a_{14} a_{21} \ldots a_{n 4}} \prod_{j=1}^{n} \mathcal{D}_{a_{j 1} a_{j 2}, a_{j 3} a_{j 4}}^{\boldsymbol{p}_{j}} \mathcal{M}
$$

where $\left(\epsilon_{i}, V_{i}\right)$ stand for the collection of the vertex factors and polarization vectors, $\boldsymbol{p}_{j}$ is sum of some bulk to boundary momenta depending on the topology of the diagram, and $\mathcal{M}$ is the scalar factor of the amplitude: it is the graviton analog of the amplitude for the straight-only-graph in [38] (and $\mathcal{M}_{\text {topology }}^{(1)}$ analog of [56]).

\subsection{Bulk point integrated expressions}

In the naive order of the calculations, one needs to carry out the integration in eq. (2.11) to get the full propagator and then the radial integration to get the amplitude for the Witten diagram. The integration in the propagator comes from the equation of motion as derived in [41] and we need to carry out the bulk point integration because we go to the Fourier space of boundary coordinates only.

A clearer approach is to interchange the order of integrations as one needs same bulkpoint integrated quantities for any tree level diagram; therefore, one can carry out bulk point integration once and for all as we did in our previous paper for gluons. In accordance with that paper's convention, we define the bulk-point integrated objects $\mathcal{K} \mathcal{K} \mathcal{K}, \mathcal{K} \mathcal{K} \mathcal{J}$, $\mathcal{K} \mathcal{J} \mathcal{J}$, and $\mathcal{J} \mathcal{J} \mathcal{J}$ as follows:

$$
\begin{aligned}
\mathcal{K} \mathcal{K} \mathcal{K}\left(k_{1}, k_{2}, k_{3}\right):= & \int_{0}^{\infty} \frac{d z}{z^{d+1}} z^{8}\left(\sqrt{\frac{2}{\pi}} z^{-2}\left(k_{1} z\right)^{\frac{d}{2}} K_{\frac{d}{2}}\left(k_{1} z\right)\right)\left(\sqrt{\frac{2}{\pi}} z^{-2}\left(k_{2} z\right)^{\frac{d}{2}} K_{\frac{d}{2}}\left(k_{2} z\right)\right) \\
& \times\left(\sqrt{\frac{2}{\pi}} z^{-2}\left(k_{3} z\right)^{\frac{d}{2}} K_{\frac{d}{2}}\left(k_{3} z\right)\right), \\
\mathcal{K} \mathcal{K} \mathcal{J}\left(k_{1}, k_{2}, k_{3}\right):= & \int_{0}^{\infty} \frac{d z}{z^{d+1}} z^{8}\left(\sqrt{\frac{2}{\pi}} z^{-2}\left(k_{1} z\right)^{\frac{d}{2}} K_{\frac{d}{2}}\left(k_{1} z\right)\right)\left(\sqrt{\frac{2}{\pi}} z^{-2}\left(k_{2} z\right)^{\frac{d}{2}} K_{\frac{d}{2}}\left(k_{2} z\right)\right) \\
& \times\left(z^{-2}(z)^{\frac{d}{2}} J_{\frac{d}{2}}\left(k_{3} z\right)\right), \\
\mathcal{K} \mathcal{J} \mathcal{J}\left(k_{1}, k_{2}, k_{3}\right):= & \int_{0}^{\infty} \frac{d z}{z^{d+1}} z^{8}\left(\sqrt{\frac{2}{\pi}} z^{-2}\left(k_{1} z\right)^{\frac{d}{2}} K_{\frac{d}{2}}\left(k_{1} z\right)\right)\left(z^{-2}(z)^{\frac{d}{2}} J_{\frac{d}{2}}\left(k_{2} z\right)\right)\left(z^{-2}(z)^{\frac{d}{2}} J_{\frac{d}{2}}\left(k_{3} z\right)\right), \\
\mathcal{J} \mathcal{J} \mathcal{J}\left(k_{1}, k_{2}, k_{3}\right):= & \int_{0}^{\infty} \frac{d z}{z^{d+1}} z^{8}\left(z^{-2}(z)^{\frac{d}{2}} J_{\frac{d}{2}}\left(k_{1} z\right)\right)\left(z^{-2}(z)^{\frac{d}{2}} J_{\frac{d}{2}}\left(k_{2} z\right)\right)\left(z^{-2}(z)^{\frac{d}{2}} J_{\frac{d}{2}}\left(k_{3} z\right)\right),
\end{aligned}
$$


where $z^{8}$ factor comes from the contraction with the three point vertex $\mathcal{V}_{\boldsymbol{k}_{1}, \boldsymbol{k}_{2}, \boldsymbol{k}_{3}}^{i j k m n}$. From now on, we will use only use the remaining tensor part $V_{\boldsymbol{k}_{1}, \boldsymbol{k}_{2}, \boldsymbol{k}_{3}}^{i j k \boldsymbol{m} n}$ in the expressions.

In terms of these objects, the amplitude for a generic tree level Witten diagram in eq. (2.15) takes the form ${ }^{10}$

$\mathcal{A}_{\mathrm{Witten}}=\left(\epsilon_{i}, V_{i}\right)^{a_{11} a_{12} \ldots a_{14} a_{21} \ldots a_{n 4}} \prod_{j=1}^{n} \mathcal{D}_{a_{j 1} a_{j 2}, a_{j 3} a_{j 4}}^{p_{j}} \int_{0}^{\infty} \prod_{k=1}^{n} \frac{\omega_{k} d \omega_{k}}{p_{k}^{2}+\omega_{k}^{2}-i \epsilon} f(\mathcal{K} \mathcal{K} \mathcal{J}, \mathcal{K} \mathcal{J} \mathcal{J}, \mathcal{J} \mathcal{J} \mathcal{J})$,

where the function $f$ depends on the topology of the diagram. Here $f(\mathcal{K} \mathcal{K} \mathcal{J}, \mathcal{K} \mathcal{J} \mathcal{J}, \mathcal{J} \mathcal{J})$ depends on $k_{1} \cdots k_{m}$ and $\omega_{1} \cdots \omega_{n}$ where $m$ is the number of external legs and $n$ is the number of bulk to bulk propagators. Likewise, $\boldsymbol{p}_{j}$ and $p_{k}$ are combinations of external momenta depending on the topology. ${ }^{11}$

As a non-trivial example, we can write the expression for the star-triangle six point diagram as

$$
\begin{aligned}
& \mathcal{A}_{\text {star-triangle }}=\left(\epsilon_{i_{1} j_{1}}^{1} \epsilon_{k_{1} l_{1}}^{2} V_{\boldsymbol{k}_{1}, \boldsymbol{k}_{2},-\boldsymbol{k}_{12}}^{i_{1} j_{1} k_{1} l_{1} m_{1} n_{1}}\right)\left(\epsilon_{i_{2} j_{2}}^{3} \epsilon_{k_{2} l_{2}}^{4} V_{\boldsymbol{k}_{3}, \boldsymbol{k}_{4},-\boldsymbol{k}_{\underline{3}}}^{i_{2} j_{2} k_{2} l_{2} m_{2} n_{2}}\right)\left(\epsilon_{i_{3} j_{3}}^{5} \epsilon_{k_{3} l_{3}}^{6} V_{\boldsymbol{k}_{5}, \boldsymbol{k}_{6},-\boldsymbol{k}_{\underline{5}}}^{i_{3} j_{3} k_{3} l_{3} m_{3} n_{3}}\right) \\
& \times V_{\boldsymbol{k}_{\underline{12}}, \boldsymbol{k}_{\underline{34}}, \boldsymbol{k}_{\underline{56}}}^{s_{1} t_{1} s_{2} t_{2} s_{3} t_{3}} \mathcal{D}_{m_{1} n_{1} s_{1} t_{1}}^{\boldsymbol{k}_{12}} \mathcal{D}_{m_{2} n_{2} s_{2} t_{2}}^{\boldsymbol{k}_{34}} \mathcal{D}_{m_{3} n_{3} s_{3} t_{3}}^{\boldsymbol{k}_{5}} \int_{0}^{\infty} \frac{\omega_{1} d \omega_{1}}{k_{\underline{12}}^{2}+\omega_{1}^{2}-i \epsilon} \int_{0}^{\infty} \frac{\omega_{2} d \omega_{2}}{k_{\underline{34}}^{2}+\omega_{2}^{2}-i \epsilon} \\
& \times \int_{0}^{\infty} \frac{\omega_{3} d \omega_{3}}{k_{56}^{2}+\omega_{3}^{2}-i \epsilon} \mathcal{K} \mathcal{K} \mathcal{J}\left(k_{1}, k_{2}, \omega_{1}\right) \mathcal{K} \mathcal{K} \mathcal{J}\left(k_{3}, k_{4}, \omega_{2}\right) \mathcal{K} \mathcal{K} \mathcal{J}\left(k_{5}, k_{6}, \omega_{3}\right) \mathcal{J} \mathcal{J} \mathcal{J}\left(\omega_{1}, \omega_{2}, \omega_{3}\right),
\end{aligned}
$$

where we are using the shorthand notation of [56]:

$$
\begin{aligned}
\underline{k_{i_{11} i_{12} \ldots i_{1 n_{1}}}} \underline{i_{21} i_{22} \ldots i_{2 n_{2}} \ldots i_{m 1} i_{m 2} \ldots i_{m n}} j_{1} j_{2} \ldots j_{p} & :=\sum_{a=1}^{m}\left|\sum_{b=1}^{n_{a}} \boldsymbol{k}_{i_{a b}}\right|+\sum_{c=1}^{p}\left|\boldsymbol{k}_{j_{c}}\right|, \\
\boldsymbol{k}_{i_{1} i_{2} \ldots i_{n}} & :=\boldsymbol{k}_{i_{1}}+\boldsymbol{k}_{i_{2}}+\cdots+\boldsymbol{k}_{i_{n}},
\end{aligned}
$$

thus, e.g. $k_{123}=\left|\boldsymbol{k}_{1}\right|+\left|\boldsymbol{k}_{2}\right|+\left|\boldsymbol{k}_{3}\right|, k_{123} \underline{456}=\left|\boldsymbol{k}_{1}\right|+\left|\boldsymbol{k}_{2}+\boldsymbol{k}_{3}\right|+\left|\boldsymbol{k}_{4}+\boldsymbol{k}_{5}\right|+\left|\boldsymbol{k}_{6}\right|, \boldsymbol{k}_{12}=\boldsymbol{k}_{1}+\boldsymbol{k}_{2}$. Even though $\boldsymbol{k}_{12}$ can also be used to label $12^{\text {th }}$ momentum in a generic calculation, there will not be room for confusion in this paper.

In this paper, we are focusing on $d=3$, and we will need the explicit expressions for $\mathcal{K} \mathcal{K} \mathcal{J}$ and $\mathcal{K} \mathcal{J} \mathcal{J}$ only:

$$
\begin{aligned}
\mathcal{K} \mathcal{K} \mathcal{J}\left(k_{1}, k_{2}, k_{3}\right) & =\frac{\sqrt{\frac{2}{\pi}} k_{3}^{3 / 2}\left(k_{1}^{2}+4 k_{2} k_{1}+k_{2}^{2}+k_{3}^{2}\right)}{\left(\left(k_{1}+k_{2}\right)^{2}+k_{3}^{2}\right)^{2}}, \\
\mathcal{K} \mathcal{J} \mathcal{J}\left(k_{1}, k_{2}, k_{3}\right) & =\frac{32 k_{1}^{3}\left(k_{2} k_{3}\right)^{3 / 2}}{\pi\left(k_{1}^{4}+2\left(k_{2}^{2}+k_{3}^{2}\right) k_{1}^{2}+\left(k_{2}^{2}-k_{3}^{2}\right)^{2}\right)^{2}} .
\end{aligned}
$$

\footnotetext{
${ }^{10}$ The factor $\mathcal{K} \mathcal{K} \mathcal{K}$ only appears in three point amplitude hence we ignored the dependence of $\mathcal{A}_{\text {Witten }}$ on $\mathcal{K} \mathcal{K} \mathcal{K}$ in eq. (2.17). Likewise, the function $f$ may depend on other bulk point integrated objects, e.g. $\mathcal{K} \mathcal{K} \mathcal{J} \mathcal{J}$, if we allow interactions beyond cubic vertices.

${ }^{11}$ Throughout the paper, we refer to expressions for particular Witten diagrams as amplitudes for those diagrams for brevity. However, one should note that the full amplitudes are sums over various channels and relevant contact terms.
} 


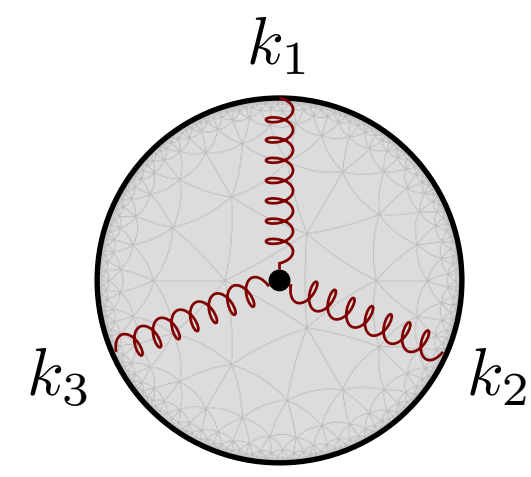

Figure 1. The three point graviton amplitude.

\section{Amplitudes}

\subsection{A basic Witten diagram: three point function}

As a warm-up, we will compute the three point amplitude. It is a useful exercise as all the dynamical data in a CFT is actually contained solely in three point correlation functions $[43,77] .{ }^{12}$ With the ingredients in eq. (2.3), we can calculate the momentum space Witten diagram shown in figure 1:

$$
\mathcal{A}_{3}=\int_{0}^{\infty} \frac{d z}{z^{d+1}} h_{i j}\left(\boldsymbol{k}_{1}, z\right) h_{k l}\left(\boldsymbol{k}_{2}, z\right) h_{m n}\left(\boldsymbol{k}_{3}, z\right) \mathcal{V}_{\boldsymbol{k}_{1}, \boldsymbol{k}_{2}, \boldsymbol{k}_{3}}^{i j k l m n}
$$

which then reads

$$
\mathcal{A}_{3}=\epsilon_{i j}^{1} \epsilon_{k l}^{2} \epsilon_{m n}^{3} V_{\boldsymbol{k}_{1}, \boldsymbol{k}_{2}, \boldsymbol{k}_{3}}^{i j k l m n}\left(\frac{2}{\pi}\right)^{3 / 2}\left(k_{1} k_{2} k_{3}\right)^{\frac{d}{2}} \int_{0}^{\infty} z^{\frac{d+2}{2}} d z K_{\frac{d}{2}}\left(k_{1} z\right) K_{\frac{d}{2}}\left(k_{2} z\right) K_{\frac{d}{2}}\left(k_{3} z\right) .
$$

In $d=3$, the integration becomes

$$
\begin{aligned}
& \left(\frac{2}{\pi} k_{1} k_{2} k_{3}\right)^{3 / 2} \int z^{\frac{5}{2}} d z K_{\frac{3}{2}}\left(k_{1} z\right) K_{\frac{3}{2}}\left(k_{2} z\right) K_{\frac{3}{2}}\left(k_{3} z\right)=-\frac{k_{1} k_{2} k_{3} z e^{\left(-k_{1}-k_{2}-k_{3}\right) z}}{k_{1}+k_{2}+k_{3}} \\
& \quad-\frac{k_{1} k_{2} k_{3} e^{\left(-k_{1}-k_{2}-k_{3}\right) z}}{\left(k_{1}+k_{2}+k_{3}\right)^{2}}-\frac{\left(k_{2} k_{3}+k_{1} k_{2}+k_{1} k_{3}\right) e^{\left(-k_{1}-k_{2}-k_{3}\right) z}}{k_{1}+k_{2}+k_{3}}-\frac{e^{\left(-k_{1}-k_{2}-k_{3}\right) z}}{z}
\end{aligned}
$$

which yields the regularized result

$$
\mathcal{A}_{3}=\epsilon_{i j}^{1} \epsilon_{k l}^{2} \epsilon_{m n}^{3} V_{\boldsymbol{k}_{1}, \boldsymbol{k}_{2}, \boldsymbol{k}_{3}}^{i j k l m n}\left[\frac{k_{1} k_{2} k_{3}}{\left(k_{1}+k_{2}+k_{3}\right)^{2}}+\frac{k_{2} k_{3}+k_{1} k_{2}+k_{1} k_{3}}{k_{1}+k_{2}+k_{3}}+\left(k_{1}+k_{2}+k_{3}\right)\right] .
$$

\footnotetext{
${ }^{12}$ We will analyze three point function dual to CFT correlator of stress tensors only; to access full dynamical data, one of course needs to consider three point function of all multitrace operators built out of the stress tensor.
} 


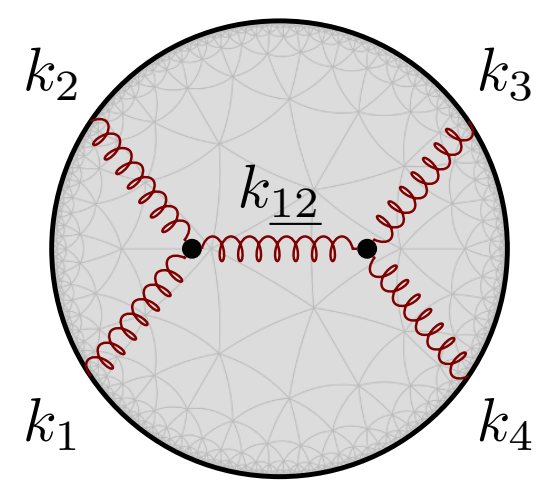

Figure 2. The four point graviton amplitude.

This result also appears in [36] where instead of $z$ integral the details appears from the computation of the time integral. Finally, we also know that,

$$
\begin{aligned}
\epsilon_{i j}^{1} \epsilon_{k l}^{2} \epsilon_{m n}^{3} V_{\boldsymbol{k}_{1}, \boldsymbol{k}_{2}, \boldsymbol{k}_{3}}^{i j k l m n}= & \epsilon_{i j}^{1} \epsilon_{k l}^{2} \epsilon_{m n}^{3}\left(\frac{\left(\boldsymbol{k}_{2}\right)^{i}\left(\boldsymbol{k}_{3}\right)^{j} \eta^{k m} \eta^{l n}}{4}-\frac{\left(\boldsymbol{k}_{2}\right)^{i}\left(\boldsymbol{k}_{3}\right)^{k} \eta^{j m} \eta^{l n}}{2}\right) \\
& +\epsilon_{i j}^{1} \epsilon_{k l}^{2} \epsilon_{m n}^{3}\left(\frac{\left(\boldsymbol{k}_{3}\right)^{k}\left(\boldsymbol{k}_{1}\right)^{l} \eta^{m i} \eta^{n j}}{4}-\frac{\left(\boldsymbol{k}_{3}\right)^{k}\left(\boldsymbol{k}_{1}\right)^{m} \eta^{l i} \eta^{n j}}{2}\right) \\
& +\epsilon_{i j}^{1} \epsilon_{k l}^{2} \epsilon_{m n}^{3}\left(\frac{\left(\boldsymbol{k}_{1}\right)^{m}\left(\boldsymbol{k}_{2}\right)^{n} \eta^{i k} \eta^{j l}}{4}-\frac{\left(\boldsymbol{k}_{1}\right)^{m}\left(\boldsymbol{k}_{2}\right)^{i} \eta^{n k} \eta^{j l}}{2}\right) \\
= & \frac{1}{4}\left[\left(\boldsymbol{k}_{2} \epsilon_{1} \boldsymbol{k}_{3}\right)\left(\epsilon_{2} \epsilon_{3}\right)+\left(\boldsymbol{k}_{3} \epsilon_{2} \boldsymbol{k}_{1}\right)\left(\epsilon_{3} \epsilon_{1}\right)+\left(\boldsymbol{k}_{1} \epsilon_{3} \boldsymbol{k}_{2}\right)\left(\epsilon_{1} \epsilon_{2}\right)\right] \\
& -\frac{1}{2}\left[\left(\boldsymbol{k}_{2} \epsilon_{1} \epsilon_{3} \epsilon_{2} \boldsymbol{k}_{3}\right)+\left(\boldsymbol{k}_{3} \epsilon_{2} \epsilon_{1} \epsilon_{3} \boldsymbol{k}_{1}\right)+\left(\boldsymbol{k}_{1} \epsilon_{3} \epsilon_{2} \epsilon_{1} \boldsymbol{k}_{2}\right)\right]
\end{aligned}
$$

with the shorthand notation $\left(\boldsymbol{k}_{a} \epsilon_{b_{1}} \ldots \epsilon_{b_{n}} \boldsymbol{k}_{b}\right) \equiv\left(\boldsymbol{k}_{a}\right)^{i_{1}} \epsilon_{i_{1} i_{2}}^{b_{1}} \eta^{i_{2} i_{3}} \epsilon_{i_{3} i_{4}}^{b_{2}} \eta^{i_{4} i_{5}} \ldots \epsilon_{i_{2 n-1} i_{2 n}}^{b_{n}}\left(\boldsymbol{k}_{a}\right)^{i_{2 n}}$ and $\left(\epsilon_{a} \epsilon_{b}\right) \equiv \epsilon_{i j}^{a} \epsilon_{k l}^{b} \eta^{i k} \eta^{j l}$.

Therefore, the amplitude associated with the three point diagram reads explicitly as

$$
\begin{aligned}
\mathcal{A}_{3}= & {\left[\frac{1}{4}\left(\left(\boldsymbol{k}_{2} \epsilon_{1} \boldsymbol{k}_{3}\right)\left(\epsilon_{2} \epsilon_{3}\right)+\left(\boldsymbol{k}_{3} \epsilon_{2} \boldsymbol{k}_{1}\right)\left(\epsilon_{3} \epsilon_{1}\right)+\left(\boldsymbol{k}_{1} \epsilon_{3} \boldsymbol{k}_{2}\right)\left(\epsilon_{1} \epsilon_{2}\right)\right)\right.} \\
& \left.-\frac{1}{2}\left(\left(\boldsymbol{k}_{2} \epsilon_{1} \epsilon_{3} \epsilon_{2} \boldsymbol{k}_{3}\right)+\left(\boldsymbol{k}_{3} \epsilon_{2} \epsilon_{1} \epsilon_{3} \boldsymbol{k}_{1}\right)+\left(\boldsymbol{k}_{1} \epsilon_{3} \epsilon_{2} \epsilon_{1} \boldsymbol{k}_{2}\right)\right)\right] \\
\times & {\left[\frac{k_{1} k_{2} k_{3}}{\left(k_{1}+k_{2}+k_{3}\right)^{2}}+\frac{k_{2} k_{3}+k_{1} k_{2}+k_{1} k_{3}}{k_{1}+k_{2}+k_{3}}+\left(k_{1}+k_{2}+k_{3}\right)\right] . }
\end{aligned}
$$

\subsection{Four point function}

In this section, we will step by step compute the amplitude associated with the $s$-channel four point Witten diagram shown in figure $2 .{ }^{13}$ For this topology, eq. (2.17) becomes

$$
\begin{aligned}
\mathcal{A}_{\text {four point }}= & \left(\epsilon_{i_{1} j_{1}}^{1} \epsilon_{k_{1} l_{1}}^{2} V_{\boldsymbol{k}_{1}, \boldsymbol{k}_{2}, \boldsymbol{k}_{\underline{12}}}^{i_{1} j_{1} k_{1} l_{1} m_{1} n_{1}}\right)\left(\epsilon_{i_{2} j_{2}}^{3} \epsilon_{k_{2} l_{2}}^{4} V_{\boldsymbol{k}_{3}, \boldsymbol{k}_{4},-\boldsymbol{k}_{12}}^{i_{2} j_{2} k_{2} l_{2} m_{2} n_{2}}\right) \\
& \times \mathcal{D}_{m_{1} n_{1} m_{2} n_{2}}^{\boldsymbol{k}_{12}} \int_{0}^{\infty} \frac{\omega d \omega}{k_{\underline{12}}^{2}+\omega^{2}-i \epsilon} \mathcal{K} \mathcal{K} \mathcal{J}\left(k_{1}, k_{2}, \omega\right) \mathcal{K} \mathcal{K} \mathcal{J}\left(k_{3}, k_{4}, \omega\right) .
\end{aligned}
$$

\footnotetext{
${ }^{13}$ One can write down expressions associated with other channels in a similar fashion.
} 
We first focus on the scalar factor. From eq. (2.16), it reads as

$$
\begin{aligned}
\mathcal{M}_{\text {four point }} & :=\int_{0}^{\infty} \frac{\omega d \omega}{k_{12}^{2}+\omega^{2}-i \epsilon} \mathcal{K} \mathcal{K} \mathcal{J}\left(k_{1}, k_{2}, \omega\right) \mathcal{K} \mathcal{K} \mathcal{J}\left(k_{3}, k_{4}, \omega\right) \\
& =\int_{0}^{\infty} d \omega \frac{2 \omega^{4}\left(k_{1}^{2}+k_{2}^{2}+4 k_{1} k_{2}+\omega^{2}\right)\left(k_{3}^{2}+k_{4}^{2}+4 k_{3} k_{4}+\omega^{2}\right)}{\pi\left(\left(k_{1}+k_{2}\right)^{2}+\omega^{2}\right)^{2}\left(\left(k_{3}+k_{4}\right)^{2}+\omega^{2}\right)^{2}\left(k_{\underline{12}}^{2}+\omega^{2}-i \epsilon\right)}
\end{aligned}
$$

We can carry out this integration ${ }^{14}$ to find

$$
\begin{aligned}
\mathcal{M}_{\text {four point }}= & \frac{1}{\left(-k_{\underline{12}}+k_{1}+k_{2}\right)^{2}\left(k_{\underline{12}}+k_{1}+k_{2}\right)^{2}}\left(\frac{2 k_{1} k_{2} k_{3}\left(k_{1}+k_{2}+k_{3}\right)\left(-k_{\underline{12}}+k_{1}+k_{2}\right)\left(k_{\underline{12}}+k_{1}+k_{2}\right)}{\left(k_{1}+k_{2}+k_{3}+k_{4}\right)^{3}}\right. \\
& -\frac{k_{3} k_{\underline{12}}\left(k_{\underline{12}}+k_{3}\right)\left(-k_{12}^{2}+k_{1}^{2}+4 k_{2} k_{1}+k_{2}^{2}\right)}{\left(k_{\underline{12}}+k_{3}+k_{4}\right)^{2}}-\frac{\left(-k_{\underline{12}}^{2}+k_{1}^{2}+4 k_{2} k_{1}+k_{2}^{2}\right)\left(k_{\underline{12}}^{2}+k_{3}^{2}\right)}{k_{\underline{12}}+k_{3}+k_{4}} \\
& +\frac{k_{1}^{2}\left(-k_{\underline{12}}^{2}+6 k_{2}^{2}+k_{3}^{2}\right)-\left(k_{2}^{2}+k_{3}^{2}\right)\left(k_{\underline{12}}-k_{2}\right)\left(k_{\underline{12}}+k_{2}\right)+k_{1}^{4}+4 k_{2} k_{1}^{3}+4 k_{2}\left(k_{2}^{2}+k_{3}^{2}\right) k_{1}}{k_{1}+k_{2}+k_{3}+k_{4}} \\
& +\frac{\left(k_{1}+k_{2}\right) k_{3}^{2}\left(-k_{\underline{12}}^{2}+k_{1}^{2}+4 k_{2} k_{1}+k_{2}^{2}\right)+k_{3}\left(-k_{1}^{2}\left(k_{\underline{12}}^{2}-6 k_{2}^{2}\right)-k_{2}^{2} k_{\underline{12}}^{2}+k_{1}^{4}+4 k_{2} k_{1}^{3}+4 k_{2}^{3} k_{1}+k_{2}^{4}\right)}{\left(k_{1}+k_{2}+k_{3}+k_{4}\right)^{2}} \\
& \left.+\frac{k_{1} k_{2}\left(k_{1}+k_{2}\right)\left(-k_{\underline{12}}+k_{1}+k_{2}\right)\left(k_{\underline{12}}+k_{1}+k_{2}\right)}{\left(k_{1}+k_{2}+k_{3}+k_{4}\right)^{2}}\right) .
\end{aligned}
$$

The result seems rather complicated; however it can be put in a simpler and more intuitive form. For this, we need to make use of the symmetries of the four point exchange diagram. As we can see from figure 2 , the scalar factor $\mathcal{M}_{\text {four point }}$ should have the symmetries $k_{1} \leftrightarrow k_{2}, k_{3} \leftrightarrow k_{4}$, and $\left\{k_{1}, k_{2}\right\} \leftrightarrow\left\{k_{3}, k_{4}\right\}$, hence we can change variables to those invariant under these interchanges:

$$
\begin{aligned}
E & \equiv k_{1}+k_{2}+k_{3}+k_{4}, \\
\alpha & \equiv\left(k_{1}+k_{2}\right)\left(k_{3}+k_{4}\right), \\
\beta & \equiv k_{1} k_{2}+k_{3} k_{4}, \\
\gamma & \equiv k_{1} k_{2} k_{3} k_{4}, \\
\sigma & \equiv\left|k_{1}+k_{2}-k_{3}-k_{4}\right|, \\
\lambda & \equiv\left|k_{1} k_{2}-k_{3} k_{4}\right| .
\end{aligned}
$$

Of course, not all of these parameters are independent; in fact, we have the relations $E^{2}-4 \alpha=\sigma^{2}$ and $\beta^{2}-4 \gamma=\lambda^{2}$. With these parameters, the scalar factor now reads as

$$
\begin{aligned}
\mathcal{M}_{\text {four point }}= & \frac{1}{\left(E k_{\underline{12}}+k_{\underline{12}}^{2}+\alpha\right)^{2}}\left(\frac{2 \gamma\left(k_{\underline{12}}^{2}+\alpha\right)}{E^{3}}-\frac{-8 \gamma k_{\underline{12}}+\lambda \sigma k_{\underline{12}}^{2}+\alpha \lambda \sigma}{2 E^{2}}\right. \\
& \left.+\frac{k_{\underline{12}}^{2}(2 \alpha+3 \beta)-2 \lambda \sigma k_{\underline{12}}+\alpha(2 \alpha+\beta)}{2 E}+E k_{\underline{12}}^{2}+k_{\underline{12}}\left(k_{\underline{12}}^{2}+2 \alpha+\beta\right)\right) .
\end{aligned}
$$

\footnotetext{
${ }^{14} \mathrm{As}$ advocated in [56], we use residue theorem to efficiently compute these symbolic integrations. We numerically verified this result, as well as the other symbolic integrations we compute below.
} 


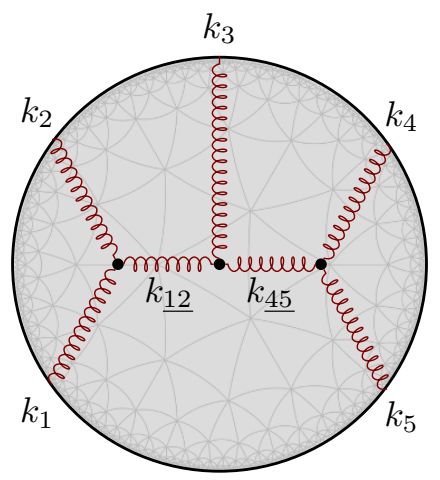

Figure 3. Five point graviton amplitude.

By acting our differential operator in eq. (2.13),

$$
\begin{aligned}
& \mathcal{M}_{m_{1} n_{1} m_{2} n_{2}}^{\text {four point }}:=-2 i \mathcal{D}_{m_{1} n_{1} m_{2} n_{2}}^{\boldsymbol{k}_{12}} \mathcal{M}_{\text {four point }}= \\
& \frac{\lambda \sigma\left(k_{\underline{12}}^{2} \mathcal{P}_{m_{1} n_{1}, m_{2} n_{2}}^{(2) k_{12}}-\alpha\left(\frac{\alpha\left(k_{\underline{12}}^{2}+\alpha\right) \mathcal{P}_{m_{1} n_{1}, m_{2} n_{2}}^{(0) k_{12}}}{\left(k_{\underline{12}}\left(k_{\underline{12}}+E\right)+\alpha\right)^{2}}+\mathcal{P}_{m_{1} n_{1}, m_{2} n_{2}}^{(1) k_{12}}\right)\right)}{2 \alpha^{2}}+\frac{4 \gamma k_{\underline{12}} \mathcal{P}_{m_{1} n_{1}, m_{2} n_{2}}^{(0) k_{12}}}{\left(k_{\underline{\underline{12}}}\left(k_{\underline{12}}+E\right)+\alpha\right)^{2}} \\
& +\frac{k_{12}\left(k_{\underline{12}}^{2}+2 \alpha+\beta\right) \mathcal{P}_{m_{1} n_{1}, m_{2} n_{2}}^{(0) k_{12}}}{\left(k_{\underline{12}}\left(k_{\underline{12}}+E\right)+\alpha\right)^{2}}+E\left(\frac{k_{\underline{12}}^{2} \mathcal{P}_{m_{1} n_{1}, m_{2} n_{2}}^{(0) k_{12}}}{\left(k_{\underline{12}}\left(k_{\underline{12}}+E\right)+\alpha\right)^{2}}-\frac{\beta k_{\underline{12}}^{2} \mathcal{P}_{m_{1} n_{1}, m_{2} n_{2}}^{(2) k_{12}}}{2 \alpha^{3}}\right) \\
& +\frac{2 \gamma\left(\alpha\left(\frac{\alpha\left(k_{\underline{12}}^{2}+\alpha\right) \mathcal{P}_{m_{1} n_{1}, m_{2} n_{2}}^{(0) k_{12}}}{\left(k_{\underline{12}}\left(k_{\underline{12}}+E\right)+\alpha\right)^{2}}+\mathcal{P}_{m_{1} n_{1}, m_{2} n_{2}}^{(1) k_{12}}\right)-k_{\underline{12}}^{2} \mathcal{P}_{m_{1} n_{1}, m_{2} n_{2}}^{(2) k_{12}}\right)}{\alpha^{2} E^{3}}+\frac{\lambda \sigma \underline{k_{12}}}{\mathcal{P}_{m_{1} n_{1}, m_{2} n_{2}}^{(2) k_{12}}} \\
& \alpha^{2}\left(\frac{\alpha\left(k_{\underline{12}}^{2}(2 \alpha+3 \beta)-2 \lambda \sigma k_{\underline{12}}+\alpha(2 \alpha+\beta)\right) \mathcal{P}_{m_{1} n_{1}, m_{2} n_{2}}^{(0) k_{12}}}{\left(k_{\underline{12}}\left(k_{\underline{12}}+E\right)+\alpha\right)^{2}}+(2 \alpha+\beta) \mathcal{P}_{m_{1} n_{1}, m_{2} n_{2}}^{(1) k_{12}}\right) \\
& +\frac{+k_{\underline{12}}^{2}(\alpha(\beta-2 \alpha)-4 \gamma) \mathcal{P}_{m_{1} n_{1}, m_{2} n_{2}}^{(2) k_{12}}}{2 \alpha^{3} E},
\end{aligned}
$$

hence eq. (3.7) reads as

$$
\mathcal{A}_{\text {four point }}=\frac{i}{2}\left(\epsilon_{i_{1} j_{1}}^{1} \epsilon_{k_{1} l_{1}}^{2} V_{\boldsymbol{k}_{1}, \boldsymbol{k}_{2}, \boldsymbol{k}_{12}}^{i_{1} j_{1} k_{1} l_{1} m_{1} n_{1}}\right) \mathcal{M}_{m_{1} n_{1} m_{2} n_{2}}^{\text {four point }}\left(\epsilon_{i_{2} j_{2}}^{3} \epsilon_{k_{2} l_{2}}^{4} V_{\boldsymbol{k}_{3}, \boldsymbol{k}_{4},-\boldsymbol{k}_{12}}^{i_{2} j_{2} k_{2} l_{2} m_{2} n_{2}}\right) .
$$

\subsection{Five point function}

Here we will compute the amplitude associated with the five point Witten diagram shown in figure 3. Alongside being an interesting computational challange as it contains two bulk to bulk propagators, this Comb channel is the only diagram one can write without studying beyond cubic interactions.

Analogous to the four point diagram, we can write down the five point amplitude as

$$
\begin{aligned}
\mathcal{A}_{\text {five point }}= & \left(\epsilon_{i_{1} j_{1}}^{1} \epsilon_{k_{1} l_{1}}^{2} V_{\boldsymbol{k}_{1}, \boldsymbol{k}_{2}, \boldsymbol{k}_{12}}^{i_{1} j_{1} k_{1} l_{1} m_{1} n_{1}}\right)\left(\epsilon_{i_{3} j_{3}}^{3} V_{-\boldsymbol{k}_{12}, \boldsymbol{k}_{3},-\boldsymbol{k}_{\underline{45}}}^{p_{1} r_{1} i_{3} j_{3} p_{2} r_{2}}\right)\left(\epsilon_{i_{2} j_{2}}^{4} \epsilon_{k_{2} l_{2}}^{5} V_{\boldsymbol{k}_{4}, \boldsymbol{k}_{5}, \boldsymbol{k}_{\underline{45}}}^{i_{2} j_{j_{2} k_{2} l_{2} m_{2} n_{2}}}\right) \\
& \times \mathcal{D}_{m_{1} n_{1} p_{1} r_{1}}^{\boldsymbol{k}_{12}} \mathcal{D}_{m_{2} n_{2} p_{2} \boldsymbol{k}_{2}}^{\boldsymbol{k}_{45}} \mathcal{M}_{\text {five point }},
\end{aligned}
$$


where we define the five point scalar factor

$$
\begin{aligned}
\mathcal{M}_{\text {five point }} \equiv & \int_{0}^{\infty} \frac{\omega_{1} \omega_{2} d \omega_{1} d \omega_{2}}{\left(k_{\underline{12}}^{2}+\omega_{1}^{2}-i \epsilon\right)\left(k_{\underline{45}}^{2}+\omega_{2}^{2}-i \epsilon\right)} \\
& \times \mathcal{K} \mathcal{K} \mathcal{J}\left(k_{1}, k_{2}, \omega_{1}\right) \mathcal{K} \mathcal{J} \mathcal{J}\left(k_{3}, \omega_{1}, \omega_{2}\right) \mathcal{K} \mathcal{K} \mathcal{J}\left(k_{4}, k_{5}, \omega_{2}\right) .
\end{aligned}
$$

Such scalar factors can be calculated in a symbolic calculation program such as Mathematica, especially by taking advantage of the residue theorem as we demonstrated in our previous work. The result takes the following nice form

$$
\mathcal{M}_{\text {five point }}=\frac{\sum_{i=0}^{9} k_{3}^{i}\left(c_{i}+\left(k_{\underline{12}}-k_{\underline{45}}\right) d_{i}\right)}{2 k_{12 \underline{12}}^{2} k_{45 \underline{54}}^{2} k_{\underline{12} 3 \underline{4} \underline{k}}^{2} k_{123 \underline{45}}^{3} k_{\underline{12345}}^{3} k_{12345}^{4}} .
$$

By using an appropriate set of variables analogous to eq. (3.10) we can write the coefficients $c_{i}, d_{i}$ in a simpler form; however, they are still rather complicated so we list the explicit expressions in appendix A. The reader can also refer to the Mathematica file attached to this paper as supplementary material, where the result is presented in terms of the conventional variables $k_{i}$.

With $\mathcal{M}_{\text {five point }}$, one can straightforwardly obtain $\mathcal{A}_{\text {five point }}$ as we have demonstrated above for four point amplitude. Even though the result looks quite complicated, it can simplify in certain limits and the commutation of the limiting procedure with the act of differential operators is one of the main strengths of our formalism since it allows us to obtain final elegantly simple results in a relatively easy computation. We will discuss two such limits in the next section.

\section{Flat space and collinear limits}

Flat space limit. As mentioned in the introduction, it has been known that Witten diagrams reduce to scattering amplitudes in an appropriate flat space limit [34-37]. One of the advantages of using momentum space is that this procedure becomes almost trivial. In [34], the author derives the relation that extracts the flat space amplitude from the AdS correlators. For tree-level graviton diagrams in $\mathrm{AdS}_{4}$, the relation simply reads as

$$
\mathcal{A}_{n-\text { point }}^{\text {flat space }}=\lim _{E \rightarrow 0} \frac{E^{n-1}}{\Gamma(n-1) \prod_{i=1}^{n} k_{i}} \mathcal{A}_{n-\text { point }}
$$

for $E=k_{1}+\cdots+k_{n}$.

Let us apply this formula for the amplitudes that we examined above. In the case of three point diagram, we can immediately read off the result from eq. (3.6):

$$
\begin{aligned}
\mathcal{A}_{3}^{\text {flat space }} & =\left[\frac{1}{4}\left(\left(\boldsymbol{k}_{2} \epsilon_{1} \boldsymbol{k}_{3}\right)\left(\epsilon_{2} \epsilon_{3}\right)+\left(\boldsymbol{k}_{3} \epsilon_{2} \boldsymbol{k}_{1}\right)\left(\epsilon_{3} \epsilon_{1}\right)+\left(\boldsymbol{k}_{1} \epsilon_{3} \boldsymbol{k}_{2}\right)\left(\epsilon_{1} \epsilon_{2}\right)\right)\right. \\
& \left.-\frac{1}{2}\left(\left(\boldsymbol{k}_{2} \epsilon_{1} \epsilon_{3} \epsilon_{2} \boldsymbol{k}_{3}\right)+\left(\boldsymbol{k}_{3} \epsilon_{2} \epsilon_{1} \epsilon_{3} \boldsymbol{k}_{1}\right)+\left(\boldsymbol{k}_{1} \epsilon_{3} \epsilon_{2} \epsilon_{1} \boldsymbol{k}_{2}\right)\right)\right] .
\end{aligned}
$$


The transition to flat space limit is also quite transparent for four point diagrams. In particular, in the parameters that we introduced in eq. (3.10), eq. (4.1) becomes

$$
\mathcal{A}_{\text {four point }}^{\text {flat space }}=\lim _{E \rightarrow 0} \frac{E^{3}}{2 \gamma} \mathcal{A}_{\text {four point }}
$$

Inserting eq. (3.13) into this, we obtain the simple result

$$
\begin{aligned}
\mathcal{A}_{\text {four point }}^{\text {flat space }}= & \frac{i}{2}\left(\epsilon_{i_{1} j_{1}}^{1} \epsilon_{k_{1} l_{1}}^{2} V_{\boldsymbol{k}_{1}, \boldsymbol{k}_{2}, \boldsymbol{k}_{\underline{12}}}^{i_{1} j_{1} k_{1} l_{1} m_{1} n_{1}}\right)\left(\epsilon_{i_{2} j_{2}}^{3} \epsilon_{k_{2} l_{2}}^{4} V_{\boldsymbol{k}_{3}, \boldsymbol{k}_{4},-\boldsymbol{k}_{12}}^{i_{2} j_{2} k_{2} l_{2} m_{2} n_{2}}\right) \\
& \times\left(\frac{\mathcal{P}_{m_{1} n_{1}, m_{2} n_{2}}^{(0) k_{12}}}{k_{\underline{12}}^{2}+\alpha}+\frac{\mathcal{P}_{m_{1} n_{1}, m_{2} n_{2}}^{(1)}+\alpha}{\alpha}-\frac{k_{12}^{2} \mathcal{P}_{m_{1} n_{1}, m_{2} n_{2}}^{(2) k_{12}}}{\alpha^{2}}\right) .
\end{aligned}
$$

By inserting the explicit expressions for $\mathcal{P}$ and $V$, one can reduce the result to product of momenta and the polarization vectors; for example,

$$
\begin{aligned}
& \left(\epsilon_{i_{1} j_{1}}^{1} \epsilon_{k_{1} l_{1}}^{2} V_{\boldsymbol{k}_{1}, \boldsymbol{k}_{2}, \boldsymbol{k}_{\underline{12}}}^{i_{1} j_{1} k_{1} l_{1} m_{1} n_{1}}\right) \mathcal{P}_{m_{1} n_{1}, m_{2} n_{2}}^{(2) k_{12}}\left(\epsilon_{i_{2} j_{2}}^{3} \epsilon_{k_{2} l_{2}}^{4} V_{\boldsymbol{k}_{3}, \boldsymbol{k}_{4},-\boldsymbol{k}_{\underline{12}}}^{i_{2} j_{2} k_{2} l_{2} m_{2} n_{2}}\right)=-\frac{1}{16 k_{\underline{12}}^{4}} \\
& \times\left[\left(\boldsymbol{k}_{\underline{12}} \epsilon_{1} \boldsymbol{k}_{\underline{12}}\right)\left(\boldsymbol{k}_{\underline{12}} \epsilon_{2} \boldsymbol{k}_{1}\right)-\left(\boldsymbol{k}_{\underline{12}} \epsilon_{2} \boldsymbol{k}_{\underline{12}}\right)\left(\boldsymbol{k}_{\underline{12}} \epsilon_{1} \boldsymbol{k}_{2}\right)+\boldsymbol{k}_{1} \cdot \boldsymbol{k}_{\underline{12}}\left(\boldsymbol{k}_{2} \cdot \boldsymbol{k}_{\underline{12}}\left(\epsilon_{1} \epsilon_{2}\right)-2\left(\boldsymbol{k}_{\underline{12}} \epsilon_{1} \epsilon_{2} \boldsymbol{k}_{\underline{12}}\right)-2\left(\boldsymbol{k}_{2} \epsilon_{1} \epsilon_{2} \boldsymbol{k}_{\underline{12}}\right)\right)\right] \\
& \times\left[\left(\boldsymbol{k}_{\underline{12}} \epsilon_{4} \boldsymbol{k}_{\underline{12}}\right)\left(\boldsymbol{k}_{\underline{12}} \epsilon_{3} \boldsymbol{k}_{4}\right)-\left(\boldsymbol{k}_{\underline{12}} \epsilon_{3} \boldsymbol{k}_{\underline{12}}\right)\left(\boldsymbol{k}_{\underline{12}} \epsilon_{4} \boldsymbol{k}_{3}\right)+\boldsymbol{k}_{3} \cdot \boldsymbol{k}_{\underline{12}}\left(\boldsymbol{k}_{4} \cdot \boldsymbol{k}_{\underline{12}}\left(\epsilon_{3} \epsilon_{4}\right)+2\left(\boldsymbol{k}_{\underline{12}} \epsilon_{3} \epsilon_{4} \boldsymbol{k}_{\underline{12}}\right)-2\left(\boldsymbol{k}_{4} \epsilon_{3} \epsilon_{4} \boldsymbol{k}_{\underline{12}}\right)\right)\right]
\end{aligned}
$$

with the shorthand notation used in eq. (3.5).

We can similarly calculate the flat space limit for five point diagram. For brevity, we will only provide the flat space limit of $\mathcal{M}_{\text {five point }}$; one can obtain the full flat space amplitude $\mathcal{A}_{\text {five point }}$ by acting with the differential operators and carrying out the relevant contractions as we demonstrated above for the four point case. We emphasize that the flat space limit commutes with the differential operators, thus this procedure is valid. The result simply reads as

$$
\mathcal{M}_{\text {five point }}^{\text {flat space }}=\lim _{k_{12345} \rightarrow 0} \frac{k_{12345}^{4}}{6 k_{1} k_{2} k_{3} k_{4} k_{5}} \mathcal{M}_{\text {five point }}=\frac{1}{\left(k_{\underline{12}}^{2}-k_{12}^{2}\right)\left(k_{\underline{123}}^{2}-k_{123}^{2}\right)} .
$$

At the level of scalar factors, the flat space limit for four point function takes a similar form

$$
\mathcal{M}_{\text {four point }}^{\text {flat space }}=\lim _{k_{1234} \rightarrow 0} \frac{k_{1234}^{3}}{2 k_{1} k_{2} k_{3} k_{4}} \mathcal{M}_{\text {four point }}=\frac{1}{k_{\underline{12}}^{2}-k_{12}^{2}} .
$$

In fact, looking at these results, we may generalize the flat space limit of scalar factors for higher point comb-like diagrams as

$$
\mathcal{M}_{n-\text { point comb-like diagram }}^{\text {flat space }}=\frac{1}{\prod_{i=1}^{n-2}\left(\left|\sum_{j=1}^{i} \boldsymbol{k}_{j}\right|^{2}-\left(\sum_{j=1}^{i}\left|\boldsymbol{k}_{j}\right|\right)^{2}\right)}
$$


Collinear limit. Another interesting limit that we may manifestly take in our formalism is the collinear limit, e.g. when vector sum of the momenta of two external legs approaches zero. In most of the cases, our relations do not provide an immediate simplification albeit they are perfectly suitable for the calculation. However, in the cases where the chosen external legs directly interact in the chosen Witten diagram, we may drastically simplify the calculation by commuting the collinear limit with the differential operator for the relevant bulk to bulk propagator.

We can see this simplification as follows. Let us consider the collinear limit of a generic Witten diagram

$$
\lim _{\boldsymbol{q} \rightarrow 0} \mathcal{A}_{\mathrm{Witten}}=\left[\lim _{\boldsymbol{q} \rightarrow 0}\left(\epsilon_{i}, V_{i}\right)^{a_{11} a_{12} \ldots a_{14} a_{21} \ldots a_{n 4}}\right] \lim _{\boldsymbol{q} \rightarrow 0} \prod_{j=1}^{n} \mathcal{D}_{a_{j 1} a_{j 2}, a_{j 3} a_{j 4}}^{\boldsymbol{p}_{j}} \mathcal{M}
$$

where $\boldsymbol{q}$ is the vanishing momentum in the chosen collinear limit, $\boldsymbol{p}_{j}$ is sum of some bulk to boundary momenta depending on the topology of the diagram and $\mathcal{M}$ is the scalar part of the amplitude. If $\mathbf{q} \in\left\{\mathbf{p}_{j}\right\}$, taking $\mathbf{p}_{n}=\mathbf{q}$, we see that

$$
\begin{aligned}
\lim _{\boldsymbol{q} \rightarrow 0} \mathcal{A}_{\mathrm{Witten}}= & -\frac{i}{2}\left(\eta_{a_{n 1} a_{n 3}} \eta_{a_{n 2} a_{n 4}}-\eta_{a_{n 1} a_{n 2}} \eta_{a_{n 3} a_{n 4}}+\eta_{a_{n 1} a_{n 4}} \eta_{a_{n 2} a_{n 3}}\right) \\
& \times\left[\lim _{\boldsymbol{q} \rightarrow 0}\left(\epsilon_{i}, V_{i}\right)^{a_{11} a_{12} \ldots a_{14} a_{21} \ldots a_{n 4}}\right] \prod_{j=1}^{n-1} \mathcal{D}_{a_{j 1} a_{j 2}, a_{j 3} a_{j 4}}^{\boldsymbol{p}_{j}}\left(\lim _{\boldsymbol{q} \rightarrow 0} \mathcal{M}\right) .
\end{aligned}
$$

This follows from the identity

$$
\lim _{\mathbf{k} \rightarrow 0} \mathcal{D}_{a b, c d}^{k} \mathcal{M}=\frac{\eta_{a c} \eta_{b d}-\eta_{a b} \eta_{b d}+\eta_{a d} \eta_{b c}}{2 i} \lim _{\mathbf{k} \rightarrow 0} \mathcal{M}
$$

which can be easily verified with eq. (2.13).

We can see this at work by analyzing the collinear limit $\mathbf{k}_{12} \rightarrow 0$ for the four point amplitude in eq. (3.7) where the drastic simplification in eq. (3.12) reads as

$$
\lim _{\boldsymbol{k}_{\underline{12}} \rightarrow 0} \mathcal{M}_{m_{1} n_{1} m_{2} n_{2}}^{\text {four point }}=-\frac{\left(4 \gamma+2 \alpha E^{2}+\beta E^{2}-E \lambda \sigma\right)}{2 \alpha E^{3}}\left(\eta_{m_{1} m_{2}} \eta_{n_{1} n_{2}}-\eta_{m_{1} n_{1}} \eta_{m_{2} n_{2}}+\eta_{m_{1} n_{2}} \eta_{m_{2} n_{1}}\right) .
$$

This leads to

$$
\lim _{\underline{k_{12}} \rightarrow 0} \mathcal{A}_{\text {four point }}=i \frac{41 k^{3}}{4096} \cos (2 \theta)\left(\epsilon_{1} \epsilon_{2}\right)\left(\epsilon_{3} \epsilon_{4}\right),
$$

where we took $\{E, \alpha, \beta, \gamma, \sigma, \lambda\} \rightarrow\left\{4 k, 4 k^{2}, 2 k^{2}, k^{4}, 0,0\right\}$ and defined $\boldsymbol{k}_{1} \cdot \boldsymbol{k}_{3}=k^{2} \cos (\theta)$ for $\left|\boldsymbol{k}_{1}\right|=\left|\boldsymbol{k}_{3}\right|=k$.

We note that flat space limit and collinear limit do not commute! For instance, taking the flat space limit after the collinear limit kills the four point amplitude whereas we get a finite result in the reverse order:

$$
\lim _{\underline{k_{12}} \rightarrow 0} \mathcal{A}_{\text {four point }}^{\text {flat space }}=i \frac{k^{2}}{128} \cos (2 \theta)\left(\epsilon_{1} \epsilon_{2}\right)\left(\epsilon_{3} \epsilon_{4}\right) .
$$




\section{Conclusion}

In this paper, we have studied graviton propagating in $\mathrm{AdS}_{4}$. As a continuation of our previous work with the gluons, we aimed to adapt and further develop the existent momentum space technology to graviton interactions, and to provide explicit results for tree level calculations. For this, we rewrote the graviton propagator in a way that reduced the complexity of the problem significantly. Explicitly, we managed to reduce the bulky part of the computation for any diagram to the calculation of a scalar factor. This rearranged form of the amplitudes may lead to a more systematic and overarching study of AdS correlators in momentum space. For instance, it is conceivable that these objects have similar recursive structures as the gauge theory correlators computed in [38]. Finding an analogous algorithmic method to compute the graviton interactions is an open problem which we hope to address in the future.

Our formalism has also practical importance in addition to being a possible step to a more systematic study of momentum space AdS correlators. In a standard calculation, one needs to carry out bulk point integrations whose number increases exponentially with the number of bulk to bulk propagators. In our settings, there is only one scalar factor per diagram, hence we need to carry out only one integration for any diagram. ${ }^{15}$ As the integrands consist of several Bessel functions, this is a rather important simplification which enables the calculations of higher point functions in practice. We demonstrated this utility by computing four and five point Witten diagrams. While these expressions are naively complicated, exploiting the symmetries of the diagrams simplifies the results considerably. We have provided a Mathematica file with the four and five point results; we hope that our results may serve as data points from which further insight can be obtained.

In the last part of our paper we discussed the flat space limit of our results. It is a nice feature of the momentum space formalism that this limit can be taken rather easily, and it is natural to wonder if such a transparent limit can help us find analogous structures between graviton and gluon to those found in flat space scattering amplitudes. For instance, KLT-like relations have been carried out in the context of cosmology [78]. Similarly, double copy like relations have been explored and are successfully realized for three point CFT correlators [79]. It is an interesting question whether these analyses can be further developed for holographic settings, and we hope that our work can assist in this direction.

Lastly, we hope our investigation can connect with cosmological bootstrap program as $\mathrm{AdS}$ and $\mathrm{dS}$ correlators are closely related. For instance, in [80, 81] authors propose a framework for the computation of late-time correlators in de Sitter space and bridge the gap with the boundary correlators in anti-de Sitter space. Moreover, it has been shown that the wave function of the universe for conformally coupled scalar theories in $d S_{4}$ can be

\footnotetext{
${ }^{15}$ Technically, there are $3^{n}$ multiple integrals of $n$ variables in standard approach where $n$ is the number of bulk to bulk propagators, and there is only one multiple integral of $n$ variables in our approach. Not all $3^{n}$ of these integrations are independent, and in fact one can reduce the number of integrations significantly by using the symmetries of the diagram. Nonetheless, we bypass any such additional analysis and reduce the number for any diagram.
} 
expressed as volumes of polytopes in the same spirit as the amplituhedron [82-84]. Since, the structures that we have calculated have a similar flavor, it is possible that they have a polytopic interpretation. It would be interesting to explore these connections.

\section{Acknowledgments}

We thank Chandramouli Chowdhury for discussions. SA is supported by NSF grant PHY1350180 and Simons Foundation grant 488651. SK was supported by DRFC Discretionary Funds from Williams College.

\section{A Scalar factor for five point diagram}

The scalar factor of the amplitude takes following form

$$
\mathcal{M}=\frac{\sum_{i=0}^{9} k_{3}^{i}\left(c_{i}+\left(k_{\underline{12}}-k_{\underline{45}}\right) d_{i}\right)}{2 k_{12 \underline{2}}^{2} k_{45 \underline{45}}^{2} k_{12}^{2} \underline{45} k_{123 \underline{5}}^{3} k_{12345}^{3} k_{12345}^{4}},
$$

where $c_{i}\left(d_{i}\right)$ carries the even (odd) part of the amplitude under the exchange $\left\{k_{1}, k_{2}\right\} \leftrightarrow\left\{k_{4}, k_{5}\right\}$. We can succinctly express these factors in parameters manifest under this symmetry:

$$
\begin{aligned}
e & \equiv k_{1}+k_{2}+k_{4}+k_{5}, \\
\alpha & \equiv\left(k_{1}+k_{2}\right)\left(k_{4}+k_{5}\right), \\
\beta & \equiv k_{1} k_{2}+k_{4} k_{5}, \\
\gamma & \equiv k_{1} k_{2} k_{4} k_{5}, \\
\sigma & \equiv\left|k_{1}+k_{2}-k_{4}-k_{5}\right|, \\
\lambda & \equiv\left|k_{1} k_{2}-k_{4} k_{5}\right|, \\
\rho & \equiv k_{\underline{12}}+k_{\underline{45}}, \\
\delta & \equiv k_{\underline{12}} k_{\underline{45}}, \\
\kappa & \equiv\left|k_{\underline{12}}-k_{\underline{45}}\right| .
\end{aligned}
$$

In these parameters $c_{i}$ and $d_{i}$ read as follows:

$$
\begin{aligned}
c_{0}= & \frac{1}{2} e \rho(2(\alpha+\delta)+e \rho)\left(4 \gamma \rho\left((\alpha-\delta)^{2}+\alpha \rho^{2}\right)+2 \delta e^{5}(\beta+\delta)+e^{4}(2 \delta(\rho(2(\alpha+\beta)+\delta)-\lambda \sigma)+\alpha \beta \rho)\right. \\
& +e^{3}\left(2 \delta\left(\alpha^{2}-2 \alpha(\beta+\delta)+2 \beta \delta+4 \gamma+\delta^{2}\right)+2 \rho^{2}(\delta(2 \alpha+\beta)+\alpha(\alpha+\beta))-\alpha \lambda \rho \sigma-4 \delta \lambda \rho \sigma\right) \\
& +e^{2} \rho\left(2 \alpha^{3}-\alpha^{2}\left(\beta+4 \delta-2 \rho^{2}\right)+\alpha\left(2 \delta(\delta-\beta)+\beta \rho^{2}+4 \gamma-2 \lambda \rho \sigma\right)+\delta(3 \beta \delta+16 \gamma-2 \lambda \rho \sigma)\right) \\
& \left.-e \rho\left(\alpha^{2} \lambda \sigma+\alpha\left(\lambda \sigma\left(\rho^{2}-2 \delta\right)-8 \gamma \rho\right)+\delta(\delta \lambda \sigma-8 \gamma \rho)\right)\right) \\
c_{1}= & \left(4 \rho\left(\alpha+\delta+e^{2}\right)+2 e(\alpha+\delta)+5 e \rho^{2}\right)\left(4 \gamma \rho\left((\alpha-\delta)^{2}+\alpha \rho^{2}\right)+2 \delta e^{5}(\beta+\delta)+e^{4}(2 \delta(\rho(2(\alpha+\beta)+\delta)-\lambda \sigma)+\alpha \beta \rho)\right. \\
& +e^{3}\left(2 \delta\left(\alpha^{2}-2 \alpha(\beta+\delta)+2 \beta \delta+4 \gamma+\delta^{2}\right)+2 \rho^{2}(\delta(2 \alpha+\beta)+\alpha(\alpha+\beta))-\alpha \lambda \rho \sigma-4 \delta \lambda \rho \sigma\right) \\
& +e^{2} \rho\left(2 \alpha^{3}-\alpha^{2}\left(\beta+4 \delta-2 \rho^{2}\right)+\alpha\left(2 \delta(\delta-\beta)+\beta \rho^{2}+4 \gamma-2 \lambda \rho \sigma\right)+\delta(3 \beta \delta+16 \gamma-2 \lambda \rho \sigma)\right) \\
& \left.-e \rho\left(\alpha^{2} \lambda \sigma+\alpha\left(\lambda \sigma\left(\rho^{2}-2 \delta\right)-8 \gamma \rho\right)+\delta(\delta \lambda \sigma-8 \gamma \rho)\right)\right)
\end{aligned}
$$


$c_{2}=\frac{1}{2}\left(2\left((\beta+3 \delta) \rho^{2}+2 \delta(4 \beta+5 \delta)\right) e^{7}+\left(9 \alpha \beta \rho+2\left(3(\alpha+\beta+2 \delta) \rho^{3}-\lambda \sigma \rho^{2}+\delta(25 \alpha+74 \beta+62 \delta) \rho-8 \delta \lambda \sigma\right)\right) e^{6}\right.$

$+\left(6(2 \alpha+\beta) \rho^{4}-6 \lambda \sigma \rho^{3}+8\left(4 \alpha^{2}+9 \beta \alpha+\gamma\right) \rho^{2}-9 \alpha \lambda \sigma \rho-148 \delta \lambda \sigma \rho+52 \delta^{3}+2 \alpha^{2} \beta+2 \delta^{2}\left(85 \rho^{2}-2 \alpha+36 \beta\right)\right.$ $\left.+2 \delta\left(3 \rho^{4}+(127 \alpha+154 \beta) \rho^{2}+12 \alpha^{2}+5 \alpha \beta+32 \gamma\right)\right) e^{5}$

$+\left(26 \rho \alpha^{3}+\left(136 \rho^{3}+15 \beta \rho+124 \delta \rho-2 \lambda \sigma\right) \alpha^{2}+\left(6 \rho^{5}+(145 \beta+322 \delta) \rho^{3}-76 \lambda \sigma \rho^{2}+36 \gamma \rho-2 \delta(55 \beta+23 \delta) \rho-42 \delta \lambda \sigma\right) \alpha\right.$ $\left.+152 \delta^{3} \rho+\delta^{2}\left(66 \rho^{3}+327 \beta \rho-32 \lambda \sigma\right)+\delta \rho\left(233 \beta \rho^{2}-296 \lambda \sigma \rho+592 \gamma\right)+2 \rho^{3}\left(\beta \rho^{2}-3 \lambda \sigma \rho+12 \gamma\right)\right) e^{4}$

$+\left(-2 \lambda \sigma \rho^{5}+57 \beta \delta \rho^{4}-215 \delta \lambda \sigma \rho^{3}+332 \beta \delta^{2} \rho^{2}-105 \delta^{2} \lambda \sigma \rho+4 \alpha^{4}+32 \delta^{4}-2 \alpha^{3}\left(-66 \rho^{2}+\beta-14 \delta\right)+10 \delta^{3}\left(8 \rho^{2}+7 \beta\right)\right.$ $+8 \gamma\left(3 \rho^{4}+143 \delta \rho^{2}+20 \delta^{2}\right)+\alpha^{2}\left(158 \rho^{4}+8 \delta \rho^{2}-33 \lambda \sigma \rho-36 \delta^{2}+8 \gamma-70 \beta \delta\right)$ $\left.+\alpha\left(107 \beta \rho^{4}-145 \lambda \sigma \rho^{3}+304 \gamma \rho^{2}-86 \delta \lambda \sigma \rho-28 \delta^{3}+2 \delta^{2}\left(58 \rho^{2}+\beta\right)+2 \delta\left(59 \rho^{4}-54 \beta \rho^{2}+84 \gamma\right)\right)\right) e^{3}$ $+\left(8 \gamma \rho^{5}+3 \delta(260 \gamma-17 \lambda \rho \sigma) \rho^{3}+32 \alpha^{4} \rho+24 \delta^{4} \rho+\delta^{2}\left(89 \beta \rho^{2}-124 \lambda \sigma \rho+420 \gamma\right) \rho+\delta^{3}(96 \beta \rho-2 \lambda \sigma)\right.$ $-2 \alpha^{3}\left(-53 \rho^{3}+8 \beta \rho+4 \delta \rho+\lambda \sigma\right)+\alpha\left(25 \beta \rho^{5}-101 \lambda \sigma \rho^{4}+2(280 \gamma+\delta(49 \delta-3 \beta)) \rho^{3}-16 \delta \lambda \sigma \rho^{2}+8 \delta(43 \gamma+\delta(2 \beta+\delta)) \rho+2 \delta^{2} \lambda \sigma\right)$ $\left.+\alpha^{2}\left(-56 \rho \delta^{2}+2\left(\lambda \sigma-6 \rho\left(\rho^{2}+8 \beta\right)\right) \delta+\rho\left(54 \rho^{4}+5 \beta \rho^{2}-92 \lambda \sigma \rho+132 \gamma\right)\right)\right) e^{2}$

$+\left(-23 \alpha \lambda \sigma \rho^{5}+2\left(10 \alpha^{3}+(3 \beta+10 \delta) \alpha^{2}+(182 \gamma+3 \beta \delta) \alpha+82 \gamma \delta\right) \rho^{4}-55 \alpha^{2} \lambda \sigma \rho^{3}-39 \delta^{2} \lambda \sigma \rho^{3}-2 \alpha \delta \lambda \sigma \rho^{3}\right.$

$+2\left((10 \alpha+13 \beta) \delta^{3}+\left(-10 \alpha^{2}+7 \beta \alpha+216 \gamma\right) \delta^{2}-\alpha\left(10 \alpha^{2}+13 \beta \alpha+4 \gamma\right) \delta+\alpha^{2}\left(10 \alpha^{2}-7 \beta \alpha+180 \gamma\right)\right) \rho^{2}-16 \alpha^{3} \lambda \sigma \rho$ $\left.-16 \delta^{3} \lambda \sigma \rho+16 \alpha \delta^{2} \lambda \sigma \rho+16 \alpha^{2} \delta \lambda \sigma \rho+8 \gamma(\alpha-\delta)^{2}(\alpha+\delta)\right) e+2 \rho\left((32 \gamma-3 \lambda \rho \sigma) \alpha^{3}+\left(\gamma\left(80 \rho^{2}-32 \delta\right)+3 \lambda \rho\left(\delta-\rho^{2}\right) \sigma\right) \alpha^{2}\right.$ $\left.\left.+\left(3 \delta \lambda \rho\left(\delta-\rho^{2}\right) \sigma-4 \gamma\left(-9 \rho^{4}+4 \delta \rho^{2}+8 \delta^{2}\right)\right) \alpha+\delta^{2}\left(48 \gamma \rho^{2}-3 \delta \lambda \sigma \rho+32 \gamma \delta\right)\right)\right)$

$c_{3}=\rho\left(\rho^{2}+\beta+2 \delta\right) e^{7}+\left(2 \rho^{4}+(5 \alpha+10 \beta+22 \delta) \rho^{2}-\lambda \sigma \rho+56 \delta^{2}+\alpha \beta+10 \alpha \delta+54 \beta \delta\right) e^{6}$

$+\left(\rho^{5}+(25 \alpha+21 \beta+31 \delta) \rho^{3}-10 \lambda \sigma \rho^{2}+\left(11 \alpha^{2}+31 \beta \alpha+160 \delta \alpha+195 \delta^{2}+4 \gamma+274 \beta \delta\right) \rho-\alpha \lambda \sigma-54 \delta \lambda \sigma\right) e^{5}$

$+\frac{1}{2}\left(31 \beta \rho^{4}+22 \delta \rho^{4}-38 \lambda \sigma \rho^{3}+384 \delta^{2} \rho^{2}+80 \gamma \rho^{2}+808 \beta \delta \rho^{2}-540 \delta \lambda \sigma \rho+8 \alpha^{3}+148 \delta^{3}+266 \beta \delta^{2}+432 \gamma \delta\right.$ $\left.+2 \alpha^{2}\left(104 \rho^{2}+5 \beta+50 \delta\right)+2 \alpha\left(28 \rho^{4}+(127 \beta+402 \delta) \rho^{2}-33 \lambda \sigma \rho+4 \gamma-2 \delta(25 \beta+32 \delta)\right)\right) e^{4}$ $+\left(66 \rho \alpha^{3}+\left(207 \rho^{3}+5 \beta \rho+52 \delta \rho-7 \lambda \sigma\right) \alpha^{2}+\left(-137 \lambda \sigma \rho^{2}+2\left(4 \rho^{4}+(85 \beta+166 \delta) \rho^{2}+66 \gamma-\delta(116 \beta+37 \delta)\right) \rho-38 \delta \lambda \sigma\right) \alpha\right.$ $\left.+140 \delta^{3} \rho+\delta^{2}\left(51 \rho^{3}+383 \beta \rho-27 \lambda \sigma\right)+4 \delta \rho\left(53 \beta \rho^{2}-91 \lambda \sigma \rho+258 \gamma\right)+\frac{1}{2} \rho^{3}\left(7 \beta \rho^{2}-25 \lambda \sigma \rho+152 \gamma\right)\right) e^{3}$ $+\frac{1}{2}\left(-5 \lambda \sigma \rho^{5}+4\left(72 \alpha^{2}+39 \beta \alpha+25 \gamma\right) \rho^{4}+8(19 \alpha+8 \beta) \delta \rho^{4}-324 \alpha \lambda \sigma \rho^{3}-352 \delta \lambda \sigma \rho^{3}+4 \alpha\left(65 \alpha^{2}-14 \beta \alpha+262 \gamma\right) \rho^{2}\right.$ $-4\left(43 \alpha^{2}+66 \beta \alpha-604 \gamma\right) \delta \rho^{2}-102 \alpha^{2} \lambda \sigma \rho-186 \delta^{2} \lambda \sigma \rho-8 \alpha \delta \lambda \sigma \rho+48 \delta^{4}+8 \alpha^{2}\left(2 \alpha^{2}-\beta \alpha+7 \gamma\right)$

$\left.+8 \alpha\left(4 \alpha^{2}-15 \beta \alpha+38 \gamma\right) \delta+4 \delta^{3}\left(21 \rho^{2}-8 \alpha+30 \beta\right)+4 \delta^{2}\left(-16 \alpha^{2}+49 \rho^{2} \alpha+2 \beta \alpha+120 \beta \rho^{2}+86 \gamma\right)\right) e^{2}$ $+\left(10 \gamma \rho^{5}-24 \delta(\lambda \rho \sigma-20 \gamma) \rho^{3}+20 \alpha^{4} \rho+8 \delta^{4} \rho+\delta^{2}\left(33 \beta \rho^{2}-72 \lambda \sigma \rho+308 \gamma\right) \rho+\delta^{3}(42 \beta \rho-4 \lambda \sigma)\right.$

$-2 \alpha^{3}\left(-26 \rho^{3}+7 \beta \rho+6 \delta \rho+2 \lambda \sigma\right)+\alpha^{2}\left(28 \rho^{5}-(9 \beta+20 \delta) \rho^{3}-64 \lambda \sigma \rho^{2}+196 \gamma \rho-14 \delta(3 \beta+2 \delta) \rho+4 \delta \lambda \sigma\right)$ $\left.+\alpha\left(9 \beta \rho^{5}-68 \lambda \sigma \rho^{4}+4(131 \gamma+\delta(10 \delta-3 \beta)) \rho^{3}+40 \delta \lambda \sigma \rho^{2}+2 \delta(\delta(7 \beta+6 \delta)-28 \gamma) \rho+4 \delta^{2} \lambda \sigma\right)\right) e$

$+4 \alpha^{4} \rho^{2}+\alpha^{2}\left(4 \delta \rho^{4}-15 \lambda \sigma \rho^{3}-4(\delta(\beta+\delta)-40 \gamma) \rho^{2}+6 \delta \lambda \sigma \rho-16 \gamma \delta\right)$

$+2 \alpha^{3}\left(8 \gamma+\rho\left(2 \rho^{3}-2(\beta+\delta) \rho-3 \lambda \sigma\right)\right)+\delta\left(8 \gamma\left(5 \rho^{4}+20 \delta \rho^{2}+2 \delta^{2}\right)+\delta \rho\left(-9 \lambda \sigma \rho^{2}+4 \beta \delta \rho-6 \delta \lambda \sigma\right)\right)$

$+\alpha\left(\rho\left(-7 \lambda \sigma \rho^{4}+4 \delta \lambda \sigma \rho^{2}+4 \delta^{2}(\beta+\delta) \rho+6 \delta^{2} \lambda \sigma\right)-16 \gamma\left(-10 \rho^{4}+10 \delta \rho^{2}+\delta^{2}\right)\right)$

$c_{4}=\frac{1}{2}\left(2\left(\rho^{2}-2 \delta\right) e^{7}+2 \rho\left(2 \alpha+7 \beta+8\left(\rho^{2}+\delta\right)\right) e^{6}+2\left(10 \rho^{4}+(31 \alpha+38 \beta+63 \delta) \rho^{2}-7 \lambda \sigma \rho+2 \alpha^{2}+134 \delta^{2}+7 \alpha \beta+66 \alpha \delta+152 \beta \delta\right) e^{5}\right.$ $+\left(-72 \lambda \sigma \rho^{2}+\left(6 \rho^{4}+2(69 \alpha+56 \beta+65 \delta) \rho^{2}+130 \alpha^{2}+670 \delta^{2}+176 \alpha \beta+56 \gamma+878 \alpha \delta+1037 \beta \delta\right) \rho-14 \alpha \lambda \sigma-312 \delta \lambda \sigma\right) e^{4}$ $+\left(53 \beta \rho^{4}+30 \delta \rho^{4}-84 \lambda \sigma \rho^{3}+478 \delta^{2} \rho^{2}+1101 \beta \delta \rho^{2}-1003 \delta \lambda \sigma \rho+40 \alpha^{3}+228 \delta^{3}+504 \beta \delta^{2}+8 \gamma\left(35 \rho^{2}+148 \delta\right)\right.$ $\left.+2 \alpha^{2}\left(259 \rho^{2}+9 \beta+68 \delta\right)+\alpha\left(84 \rho^{4}+(431 \beta+1362 \delta) \rho^{2}-196 \lambda \sigma \rho+56 \gamma-6 \delta(61 \beta+50 \delta)\right)\right) e^{3}$

$+\left(242 \rho \alpha^{3}-\left(-588 \rho^{3}+47 \beta \rho+124 \delta \rho+38 \lambda \sigma\right) \alpha^{2}+\left(-457 \lambda \sigma \rho^{2}+2\left(5 \rho^{4}+(180 \beta+361 \delta) \rho^{2}+374 \gamma-69 \delta(5 \beta+\delta)\right) \rho-22 \delta \lambda \sigma\right) \alpha\right.$ $\left.+268 \delta^{3} \rho+\delta^{2}\left(80 \rho^{3}+913 \beta \rho-56 \lambda \sigma\right)+\delta \rho\left(375 \beta \rho^{2}-907 \lambda \sigma \rho+3196 \gamma\right)+\rho^{3}\left(6 \beta \rho^{2}-31 \lambda \sigma \rho+312 \gamma\right)\right) e^{2}$ $+\left(-2 \lambda \sigma \rho^{5}+100 \gamma \rho^{4}-265 \delta \lambda \sigma \rho^{3}+\delta\left(29 \beta \rho^{2}+2196 \gamma\right) \rho^{2}-175 \delta^{2} \lambda \sigma \rho+20 \alpha^{4}+32 \delta^{4}+6 \delta^{3}\left(7 \rho^{2}+15 \beta\right)\right.$ $+2 \alpha^{3}\left(110 \rho^{2}-7 \beta+6 \delta\right)+8 \delta^{2}\left(39 \beta \rho^{2}+44 \gamma\right)+\alpha^{2}\left(236 \rho^{4}-2(49 \beta+121 \delta) \rho^{2}-123 \lambda \sigma \rho+144 \gamma-2 \delta(45 \beta+26 \delta)\right)$ $\left.+\alpha\left((83 \beta+90 \delta) \rho^{4}-314 \lambda \sigma \rho^{3}+2 \delta(82 \delta-101 \beta) \rho^{2}+130 \delta \lambda \sigma \rho+2(7 \beta-6 \delta) \delta^{2}+4 \gamma\left(349 \rho^{2}+4 \delta\right)\right)\right) e+16 \alpha^{4} \rho$ $-2 \alpha^{3}\left(-18 \rho^{3}+8 \beta \rho+6 \delta \rho+3 \lambda \sigma\right)+2 \alpha^{2}\left(-29 \lambda \sigma \rho^{2}-2\left(-5 \rho^{4}+(4 \beta+5 \delta) \rho^{2}-72 \gamma+\delta(6 \beta+5 \delta)\right) \rho+3 \delta \lambda \sigma\right)$ $+\alpha\left(2 \beta \rho^{5}-61 \lambda \sigma \rho^{4}+8(75 \gamma+\delta(3 \delta-\beta)) \rho^{3}+66 \delta \lambda \sigma \rho^{2}+4 \delta(\delta(4 \beta+3 \delta)-100 \gamma) \rho+6 \delta^{2} \lambda \sigma\right)$ $\left.+\delta\left(-15 \lambda \sigma \rho^{4}+16(25 \gamma+\beta \delta) \rho^{3}-60 \delta \lambda \sigma \rho^{2}+4 \delta(92 \gamma+\delta(6 \beta+\delta)) \rho-6 \delta^{2} \lambda \sigma\right)\right)$ 


$$
\begin{aligned}
& c_{5}=\frac{1}{2}\left(4 \delta^{2}\left(-4 \alpha^{2}+2 \alpha \beta+13 \alpha \rho^{2}+18 \beta \rho^{2}+40 \gamma\right)+4 \delta\left(-2 \rho^{2}\left(12 \alpha^{2}+5 \alpha \beta-98 \gamma\right)-2 \alpha(3 \alpha \beta+16 \gamma)+\rho^{4}(5 \alpha+\beta)\right)\right. \\
& +4 \alpha\left(\rho^{2}\left(17 \alpha^{2}-12 \alpha \beta+152 \gamma\right)+2 \alpha\left(\alpha^{2}-\alpha \beta+12 \gamma\right)+2 \rho^{4}(9 \alpha+\beta)\right)-50 \alpha^{2} \lambda \rho \sigma+84 \alpha \delta \lambda \rho \sigma-110 \alpha \lambda \rho^{3} \sigma \\
& +8 \delta^{3}\left(3 \beta+\rho^{2}\right)+8 \delta^{4}-66 \delta^{2} \lambda \rho \sigma-76 \delta \lambda \rho^{3} \sigma+12 e^{6}\left(\rho^{2}-2 \delta\right)+2 e^{5} \rho\left(12 \alpha+20 \beta+13 \delta+23 \rho^{2}\right) \\
& +e^{4}\left(24 \alpha^{2}+\rho^{2}(144 \alpha+139 \beta+194 \delta)+8 \alpha(5 \beta+46 \delta)+26 \delta(17 \beta+14 \delta)-40 \lambda \rho \sigma+36 \rho^{4}\right) \\
& +e^{3}\left(2 \rho\left(149 \alpha^{2}+122 \alpha \beta+674 \alpha \delta+549 \beta \delta+76 \gamma+345 \delta^{2}\right)+\rho^{3}(156 \alpha+133 \beta+138 \delta)-40 \alpha \lambda \sigma-486 \delta \lambda \sigma-117 \lambda \rho^{2} \sigma+6 \rho^{5}\right) \\
& +e^{2}\left(72 \alpha^{3}+\alpha^{2}\left(6 \beta+52 \delta+624 \rho^{2}\right)+2 \alpha\left(3 \rho^{2}(59 \beta+222 \delta)-2 \delta(119 \beta+72 \delta)+76 \gamma-140 \lambda \rho \sigma+16 \rho^{4}\right)+518 \beta \delta^{2}\right. \\
& \left.+808 \beta \delta \rho^{2}+33 \beta \rho^{4}+1512 \gamma \delta+388 \gamma \rho^{2}+196 \delta^{3}+332 \delta^{2} \rho^{2}-1030 \delta \lambda \rho \sigma+18 \delta \rho^{4}-71 \lambda \rho^{3} \sigma\right) \\
& +e\left(2 \rho^{3}\left(199 \alpha^{2}+77 \alpha \beta+202 \alpha \delta+76 \beta \delta+98 \gamma+15 \delta^{2}\right)-42 \alpha^{2} \lambda \sigma\right. \\
& +2 \rho\left(96 \alpha^{3}-\alpha^{2}(43 \beta+112 \delta)+2 \alpha\left(-103 \beta \delta+210 \gamma-8 \delta^{2}\right)+\delta\left(265 \beta \delta+1172 \gamma+64 \delta^{2}\right)\right)+\rho^{5}(\beta-4 \alpha)+52 \alpha \delta \lambda \sigma \\
& \left.\left.-354 \alpha \lambda \rho^{2} \sigma-42 \delta^{2} \lambda \sigma-576 \delta \lambda \rho^{2} \sigma-7 \lambda \rho^{4} \sigma\right)+\lambda \rho^{5} \sigma\right) \\
& c_{6}=\frac{1}{2}\left(56 \alpha^{3} \rho-8 \alpha^{2}\left(5 \beta \rho+11 \delta \rho+2 \lambda \sigma-13 \rho^{3}\right)+\alpha\left(2 \rho^{3}(7 \beta+46 \delta)+4 \delta \rho(2 \delta-19 \beta)+320 \gamma \rho+32 \delta \lambda \sigma-103 \lambda \rho^{2} \sigma-4 \rho^{5}\right)\right. \\
& +4 \delta \rho\left(\rho^{2}(5 \beta+\delta)+\delta(29 \beta+6 \delta)+180 \gamma\right)-16 \delta^{2} \lambda \sigma-151 \delta \lambda \rho^{2} \sigma+28 e^{5}\left(\rho^{2}-2 \delta\right)+2 e^{4} \rho\left(28 \alpha+28 \beta+15 \delta+31 \rho^{2}\right) \\
& +e^{3}\left(\rho^{2}(152 \alpha+125 \beta+176 \delta)+56 \alpha(\alpha+\beta)+560 \alpha \delta+354 \beta \delta+312 \delta^{2}-56 \lambda \rho \sigma+28 \rho^{4}\right) \\
& +e^{2}\left(\rho\left(334 \alpha^{2}+2 \rho^{2}(24 \alpha+33 \beta+37 \delta)+163 \alpha \beta+1232 \alpha \delta+659 \beta \delta+168 \gamma+426 \delta^{2}+2 \rho^{4}\right)-56 \alpha \lambda \sigma-438 \delta \lambda \sigma-83 \lambda \rho^{2} \sigma\right) \\
& +e\left(56 \alpha^{3}-6 \alpha^{2}\left(2 \beta+4 \delta-61 \rho^{2}\right)+\alpha\left(\rho^{2}(121 \beta+716 \delta)-20 \delta(13 \beta+6 \delta)+168 \gamma-191 \lambda \rho \sigma-24 \rho^{4}\right)+272 \beta \delta^{2}+293 \beta \delta \rho^{2}\right. \\
& \left.\left.+5 \beta \rho^{4}+952 \gamma \delta+180 \gamma \rho^{2}+88 \delta^{3}+118 \delta^{2} \rho^{2}-573 \delta \lambda \rho \sigma+4 \delta \rho^{4}-10 \lambda \rho^{3} \sigma\right)+5 \lambda \rho^{4} \sigma\right) \\
& c_{7}=8 \alpha^{3}+\alpha^{2}\left(-4 \beta-8 \delta+42 \rho^{2}\right)+\alpha\left(2 \rho^{2}(2 \beta+41 \delta)-8 \delta(3 \beta+\delta)+32 \gamma-25 \lambda \rho \sigma-8 \rho^{4}\right) \\
& +2 \delta\left(9 \beta \rho^{2}+64 \gamma\right)+4 \delta^{2}\left(7 \beta+2 \rho^{2}\right)+8 \delta^{3}-69 \delta \lambda \rho \sigma+16 e^{4}\left(\rho^{2}-2 \delta\right)+e^{3} \rho\left(32 \alpha+19 \beta+17 \delta+20 \rho^{2}\right) \\
& +e^{2}\left(32 \alpha^{2}+\frac{1}{2} \rho^{2}(62 \alpha+49 \beta+90 \delta)+19 \alpha \beta+246 \alpha \delta+77 \beta \delta+86 \delta^{2}-19 \lambda \rho \sigma+4 \rho^{4}\right) \\
& +\frac{1}{2} e\left(2 \rho\left(92 \alpha^{2}+21 \alpha \beta+316 \alpha \delta+101 \beta \delta+32 \gamma+72 \delta^{2}\right)+\rho^{3}(-34 \alpha+9 \beta+16 \delta)-38 \alpha \lambda \sigma-222 \delta \lambda \sigma-15 \lambda \rho^{2} \sigma\right)+\frac{9}{2} \lambda \rho^{3} \sigma \\
& c_{8}=\frac{5}{2}\left(8 \alpha^{2} \rho-2 \alpha\left(-14 \delta \rho+\lambda \sigma+2 \rho^{3}\right)+4 \delta \rho(\beta+\delta)-10 \delta \lambda \sigma+\lambda \rho^{2} \sigma\right)+9 e^{3}\left(\rho^{2}-2 \delta\right) \\
& +e^{2} \rho\left(18 \alpha+5 \beta+13 \delta+5 \rho^{2}\right)+e\left(18 \alpha^{2}+\alpha\left(5 \beta+118 \delta-3 \rho^{2}\right)+\frac{5}{2} \rho^{2}(\beta+4 \delta)+\delta(15 \beta+28 \delta)-5 \lambda \rho \sigma\right) \\
& c_{9}=4\left(\alpha^{2}+6 \alpha \delta+\delta\left(\delta-e^{2}\right)\right)+2 \rho^{2}\left(e^{2}-2 \alpha\right)+4 e \rho(\alpha+\delta)
\end{aligned}
$$

$$
\begin{array}{r}
d_{0}=\frac{1}{2} e \rho\left(-4 \gamma \rho \sigma\left((\alpha-\delta)^{2}+\alpha \rho^{2}\right)+2 \delta e^{6} \lambda+e^{5}(\alpha \lambda \rho-2 \delta(\sigma(\beta+\delta)-2 \lambda \rho))-e^{4}\left(\alpha\left(\beta \rho \sigma+8 \delta \lambda+4 \delta \rho \sigma-2 \lambda \rho^{2}\right)+2 \delta \rho(\sigma(2 \beta+\delta)-\lambda \rho)\right)\right. \\
-e^{3}\left(\alpha^{2}\left(2 \sigma\left(\delta+\rho^{2}\right)+3 \lambda \rho\right)+\alpha\left(2 \beta \sigma\left(\rho^{2}-2 \delta\right)-4 \delta^{2} \sigma+2 \delta \rho(9 \lambda+2 \rho \sigma)-\lambda \rho^{3}\right)+\delta\left(2 \beta \sigma\left(2 \delta+\rho^{2}\right)+8 \gamma \sigma+\delta(2 \delta \sigma-\lambda \rho)\right)\right) \\
-e^{2} \rho\left(2 \alpha^{3} \sigma+\alpha^{2}\left(8 \lambda \rho-\sigma\left(\beta+4 \delta-2 \rho^{2}\right)\right)+\alpha\left(\beta \sigma\left(\rho^{2}-2 \delta\right)+4 \gamma \sigma+2 \delta(\delta \sigma+4 \lambda \rho)\right)+\delta \sigma(3 \beta \delta+16 \gamma)\right) \\
\left.-4 e \rho\left(\alpha^{3} \lambda+\alpha^{2} \lambda\left(\rho^{2}-2 \delta\right)+\alpha\left(2 \gamma \rho \sigma+\delta^{2} \lambda\right)+2 \gamma \delta \rho \sigma\right)\right)
\end{array}
$$

$$
\begin{array}{r}
d_{1}=(e+2 \rho)\left(-4 \gamma \rho \sigma\left((\alpha-\delta)^{2}+\alpha \rho^{2}\right)+2 \delta e^{6} \lambda+e^{5}(\alpha \lambda \rho-2 \delta(\sigma(\beta+\delta)-2 \lambda \rho))\right. \\
-e^{4}\left(\alpha\left(\beta \rho \sigma+8 \delta \lambda+4 \delta \rho \sigma-2 \lambda \rho^{2}\right)+2 \delta \rho(\sigma(2 \beta+\delta)-\lambda \rho)\right) \\
-e^{3}\left(\alpha^{2}\left(2 \sigma\left(\delta+\rho^{2}\right)+3 \lambda \rho\right)+\alpha\left(2 \beta \sigma\left(\rho^{2}-2 \delta\right)-4 \delta^{2} \sigma+2 \delta \rho(9 \lambda+2 \rho \sigma)-\lambda \rho^{3}\right)+\delta\left(2 \beta \sigma\left(2 \delta+\rho^{2}\right)+8 \gamma \sigma+\delta(2 \delta \sigma-\lambda \rho)\right)\right) \\
-e^{2} \rho\left(2 \alpha^{3} \sigma+\alpha^{2}\left(8 \lambda \rho-\sigma\left(\beta+4 \delta-2 \rho^{2}\right)\right)+\alpha\left(\beta \sigma\left(\rho^{2}-2 \delta\right)+4 \gamma \sigma+2 \delta(\delta \sigma+4 \lambda \rho)\right)+\delta \sigma(3 \beta \delta+16 \gamma)\right) \\
\left.-4 e \rho\left(\alpha^{3} \lambda+\alpha^{2} \lambda\left(\rho^{2}-2 \delta\right)+\alpha\left(2 \gamma \rho \sigma+\delta^{2} \lambda\right)+2 \gamma \delta \rho \sigma\right)\right)
\end{array}
$$


$d_{2}=\frac{1}{2}\left(-4 \rho\left(3 \alpha^{3} \lambda \rho+\alpha^{2}\left(8 \gamma \sigma+3 \lambda \rho\left(\rho^{2}-2 \delta\right)\right)+\alpha\left(\gamma \sigma\left(11 \rho^{2}-16 \delta\right)+3 \delta^{2} \lambda \rho\right)+\gamma \delta \sigma\left(8 \delta+3 \rho^{2}\right)\right)-2 e^{7} \lambda \rho\right.$

$+e^{6}\left(\alpha \lambda+2 \beta \rho \sigma+20 \delta \lambda+6 \delta \rho \sigma-6 \lambda \rho^{2}\right)+e^{5}\left(\alpha\left(18 \lambda \rho-\sigma\left(\beta+2 \delta-6 \rho^{2}\right)\right)+2\left(\beta \sigma\left(3 \rho^{2}-10 \delta\right)-8 \delta^{2} \sigma+28 \delta \lambda \rho+6 \delta \rho^{2} \sigma-3 \lambda \rho^{3}\right)\right)$ $+e^{4}\left(-3 \alpha^{2} \lambda+\alpha\left(\rho\left(2 \sigma\left(6 \rho^{2}-7 \beta\right)+49 \lambda \rho\right)-2 \delta(39 \lambda+19 \rho \sigma)\right)+\delta \rho\left(2 \sigma\left(3 \rho^{2}-22 \beta\right)+49 \lambda \rho\right)+6 \beta \rho^{3} \sigma+8 \gamma \rho \sigma+\delta^{2}(5 \lambda-26 \rho \sigma)\right.$ $\left.-2 \lambda \rho^{4}\right)-e^{3}\left(2 \alpha^{3} \sigma+\alpha^{2}\left(\sigma\left(-\beta+12 \delta+16 \rho^{2}\right)+32 \lambda \rho\right)+\alpha\left(\beta \sigma\left(25 \rho^{2}-34 \delta\right)+4 \gamma \sigma-30 \delta^{2} \sigma+200 \delta \lambda \rho+58 \delta \rho^{2} \sigma-45 \lambda \rho^{3}-6 \rho^{4} \sigma\right)\right.$ $\left.+35 \beta \delta^{2} \sigma+31 \beta \delta \rho^{2} \sigma-2 \beta \rho^{4} \sigma+8 \gamma \sigma\left(10 \delta-3 \rho^{2}\right)+16 \delta^{3} \sigma-8 \delta^{2} \lambda \rho+10 \delta^{2} \rho^{2} \sigma-13 \delta \lambda \rho^{3}\right)-e^{2}\left(4 \alpha^{3}(\lambda+4 \rho \sigma)\right.$ $+\alpha^{2}\left(\rho\left(2 \sigma\left(13 \rho^{2}-4 \beta\right)+81 \lambda \rho\right)-4 \delta(2 \lambda+5 \rho \sigma)\right)+\alpha\left(2 \delta \rho\left(\sigma\left(11 \rho^{2}-20 \beta\right)+77 \lambda \rho\right)+15 \beta \rho^{3} \sigma+40 \gamma \rho \sigma+4 \delta^{2}(\lambda-2 \rho \sigma)-13 \lambda \rho^{4}\right)$ $\left.+\rho\left(\delta\left(\beta \sigma\left(48 \delta+7 \rho^{2}\right)+3 \delta(4 \delta \sigma-\lambda \rho)\right)+8 \gamma \sigma\left(23 \delta-3 \rho^{2}\right)\right)\right)$ $-e\left(2 \alpha^{3} \rho(16 \lambda+5 \rho \sigma)+\alpha^{2}\left(\rho^{2}\left(\sigma\left(10\left(\rho^{2}-2 \delta\right)-7 \beta\right)+64 \lambda \rho\right)+4 \gamma \sigma-64 \delta \lambda \rho\right)\right.$ $\left.\left.+\alpha\left(\rho\left(2 \delta \rho(16 \lambda \rho-3 \beta \sigma)+3 \beta \rho^{3} \sigma+2 \delta^{2}(16 \lambda+5 \rho \sigma)\right)-8 \gamma \sigma\left(\delta-10 \rho^{2}\right)\right)+\sigma\left(\delta \rho^{2}(13 \beta \delta+116 \gamma)+4 \gamma \delta^{2}-8 \gamma \rho^{4}\right)\right)\right)$

$d_{3}=\frac{1}{2}\left(-4\left(\alpha^{3} \rho(6 \lambda+\rho \sigma)+\alpha^{2}\left(\rho^{2}\left(\sigma\left(-\beta-2 \delta+\rho^{2}\right)+8 \lambda \rho\right)+4 \gamma \sigma-12 \delta \lambda \rho\right)+\alpha\left(2 \gamma \sigma\left(5 \rho^{2}-4 \delta\right)+\delta \rho\left(6 \delta \lambda+\delta \rho \sigma+2 \lambda \rho^{2}\right)\right)\right.\right.$ $\left.+\sigma\left(\delta \rho^{2}(\beta \delta+10 \gamma)+4 \gamma \delta^{2}-5 \gamma \rho^{4}\right)\right)-2 e^{7} \lambda+2 e^{6}\left(\sigma\left(\beta+4 \delta+\rho^{2}\right)-10 \lambda \rho\right)$ $+2 e^{5}\left(\alpha(6 \lambda+5 \rho \sigma)+\rho\left(2 \sigma\left(5 \beta+\rho^{2}\right)-19 \lambda \rho\right)+26 \delta(\lambda+\rho \sigma)\right)$ $+e^{4}\left(2 \alpha^{2} \sigma+2 \alpha\left(\sigma\left(-4 \beta-6 \delta+27 \rho^{2}\right)+50 \lambda \rho\right)-36 \beta \delta \sigma+42 \beta \rho^{2} \sigma+8 \gamma \sigma-22 \delta^{2} \sigma+130 \delta \lambda \rho+66 \delta \rho^{2} \sigma-25 \lambda \rho^{3}+2 \rho^{4} \sigma\right)$ $+e^{3}\left(4 \alpha^{2}(\rho \sigma-3 \lambda)-2 \alpha\left(\rho\left(4 \sigma\left(5 \beta-8 \rho^{2}\right)-103 \lambda \rho\right)+38 \delta(2 \lambda+\rho \sigma)\right)+2 \delta \rho\left(\sigma\left(11 \rho^{2}-19 \beta\right)+43 \lambda \rho\right)+31 \beta \rho^{3} \sigma+80 \gamma \rho \sigma\right.$ $\left.+20 \delta^{2}(\lambda-\rho \sigma)-5 \lambda \rho^{4}\right)$ $-e^{2}\left(8 \alpha^{3} \sigma+\alpha^{2}\left(2 \sigma\left(-2 \beta+4 \delta+11 \rho^{2}\right)+58 \lambda \rho\right)+2 \alpha\left(\beta \sigma\left(15 \rho^{2}-28 \delta\right)+8 \gamma \sigma-20 \delta^{2} \sigma+122 \delta \lambda \rho+34 \delta \rho^{2} \sigma-69 \lambda \rho^{3}-10 \rho^{4} \sigma\right)\right.$ $\left.+60 \beta \delta^{2} \sigma+10 \beta \delta \rho^{2} \sigma-7 \beta \rho^{4} \sigma+8 \gamma \sigma\left(20 \delta-19 \rho^{2}\right)+24 \delta^{3} \sigma-6 \delta^{2} \lambda \rho+2 \delta^{2} \rho^{2} \sigma-16 \delta \lambda \rho^{3}\right)$ $-2 e\left(2 \alpha^{3}(4 \lambda+5 \rho \sigma)+\alpha^{2}\left(\rho\left(\sigma\left(12 \rho^{2}-7 \beta\right)+40 \lambda \rho\right)-16 \delta(\lambda+\rho \sigma)\right)\right.$ $\left.\left.+\alpha\left(2 \delta \rho\left(\sigma\left(3 \rho^{2}-7 \beta\right)+28 \lambda \rho\right)+3 \beta \rho^{3} \sigma+28 \gamma \rho \sigma+2 \delta^{2}(4 \lambda+\rho \sigma)-14 \lambda \rho^{4}\right)+\rho \sigma\left(21 \beta \delta^{2}+84 \gamma \delta-50 \gamma \rho^{2}+4 \delta^{3}\right)\right)\right)$ $d_{4}=\frac{1}{2}\left(-4 \alpha^{3}(3 \lambda+2 \rho \sigma)+2 \alpha^{2}\left(\delta(12 \lambda+7 \rho \sigma)-4 \rho\left(\sigma\left(\rho^{2}-\beta\right)+3 \lambda \rho\right)\right)\right.$

$+\alpha\left(-2 \delta \rho\left(\sigma\left(\rho^{2}-2 \beta\right)+14 \lambda \rho\right)+\rho^{3}(\beta \sigma+18 \lambda \rho)-12 \gamma \rho \sigma-4 \delta^{2}(3 \lambda+\rho \sigma)\right)+\rho \sigma\left(\rho^{2}(\beta \delta+100 \gamma)-2 \delta(\delta(6 \beta+\delta)+26 \gamma)\right)$ $+2 e^{6}(\rho \sigma-7 \lambda)+2 e^{5}\left(\sigma\left(2 \alpha+7 \beta+8\left(3 \delta+\rho^{2}\right)\right)-36 \lambda \rho\right)+e^{4}\left(\alpha(60 \lambda+66 \rho \sigma)+4 \rho\left(\sigma\left(19 \beta+5 \rho^{2}\right)-21 \lambda \rho\right)+\delta(105 \lambda+166 \rho \sigma)\right)$ $+e^{3}\left(10 \alpha^{2} \sigma+\alpha\left(325 \lambda \rho-2 \sigma\left(12 \beta+15 \delta-85 \rho^{2}\right)\right)-15 \beta \delta \sigma+112 \beta \rho^{2} \sigma+56 \gamma \sigma-6 \delta^{2} \sigma+227 \delta \lambda \rho+142 \delta \rho^{2} \sigma-31 \lambda \rho^{3}+6 \rho^{4} \sigma\right)$ $+e^{2}\left(\alpha^{2}(14 \rho \sigma-13 \lambda)+\alpha\left(\rho\left(\sigma\left(124 \rho^{2}-55 \beta\right)+452 \lambda \rho\right)-2 \delta(65 \lambda+41 \rho \sigma)\right)+\delta \rho\left(\sigma\left(11 \beta+30 \rho^{2}\right)+107 \lambda \rho\right)+53 \beta \rho^{3} \sigma\right.$ $\left.+280 \gamma \rho \sigma+3 \delta^{2}(9 \lambda+2 \rho \sigma)-2 \lambda \rho^{4}\right)$ $+e\left(-10 \alpha^{3} \sigma+\alpha^{2}\left(\sigma\left(7 \beta+4\left(\delta-3 \rho^{2}\right)\right)-32 \lambda \rho\right)+\alpha\left(2 \beta \sigma\left(19 \delta-7 \rho^{2}\right)-12 \gamma \sigma+22 \delta^{2} \sigma-136 \delta \lambda \rho-38 \delta \rho^{2} \sigma+189 \lambda \rho^{3}+22 \rho^{4} \sigma\right)\right.$ $\left.\left.-45 \beta \delta^{2} \sigma+11 \beta \delta \rho^{2} \sigma+6 \beta \rho^{4} \sigma+4 \gamma \sigma\left(78 \rho^{2}-29 \delta\right)-16 \delta^{3} \sigma+4 \delta^{2} \rho^{2} \sigma+13 \delta \lambda \rho^{3}\right)\right)$

$d_{5}=\frac{1}{2}\left(-4 \alpha^{3} \sigma+2 \alpha^{2} \sigma\left(2(\beta+\delta)-\rho^{2}\right)+2 \alpha\left(\beta \sigma\left(4 \delta-\rho^{2}\right)+2\left(\delta^{2} \sigma-2 \delta \rho(4 \lambda+\rho \sigma)+2 \rho^{3}(11 \lambda+\rho \sigma)\right)\right)-12 \beta \delta^{2} \sigma+6 \beta \delta \rho^{2} \sigma\right.$ $+\beta \rho^{4} \sigma-32 \gamma \delta \sigma+196 \gamma \rho^{2} \sigma-4 \delta^{3} \sigma+2 \delta^{2} \rho^{2} \sigma+4 \delta \lambda \rho^{3}+2 e^{5}(6 \rho \sigma-20 \lambda)+e^{4}\left(2 \sigma\left(12 \alpha+20 \beta+59 \delta+23 \rho^{2}\right)-117 \lambda \rho\right)$ $+e^{3}\left(8 \alpha(20 \lambda+21 \rho \sigma)+\rho\left(\sigma\left(139 \beta+36 \rho^{2}\right)-71 \lambda \rho\right)+14 \delta(12 \lambda+19 \rho \sigma)\right)$ $+e^{2}\left(18 \alpha^{2} \sigma+\alpha\left(550 \lambda \rho-4 \sigma\left(9 \beta+10 \delta-62 \rho^{2}\right)\right)+24 \beta \delta \sigma+133 \beta \rho^{2} \sigma+152 \gamma \sigma+14 \delta^{2} \sigma+254 \delta \lambda \rho+150 \delta \rho^{2} \sigma-7 \lambda \rho^{3}+6 \rho^{4} \sigma\right)$ $+e\left(16 \alpha^{2} \rho \sigma-2 \alpha\left(\rho\left(\sigma\left(19 \beta-52 \rho^{2}\right)-227 \lambda \rho\right)+24 \delta(\lambda+\rho \sigma)\right)+2 \delta \rho\left(3 \sigma\left(5 \beta+3 \rho^{2}\right)+37 \lambda \rho\right)+33 \beta \rho^{3} \sigma+388 \gamma \rho \sigma\right.$ $\left.\left.+16 \delta^{2}(\lambda+\rho \sigma)+\lambda \rho^{4}\right)\right)$

$d_{6}=\frac{1}{2}\left(\alpha^{2}(4 \lambda+6 \rho \sigma)+\alpha\left(\rho\left(\sigma\left(32 \rho^{2}-11 \beta\right)+166 \lambda \rho\right)-4 \delta(2 \lambda+3 \rho \sigma)\right)+11 \beta \delta \rho \sigma+5 \beta \rho^{3} \sigma+180 \gamma \rho \sigma+4 \delta^{2} \lambda+6 \delta^{2} \rho \sigma+20 \delta \lambda \rho^{2}\right.$ $+4 \delta \rho^{3} \sigma+28 e^{4}(\rho \sigma-2 \lambda)+e^{3}\left(2 \sigma\left(28 \alpha+28 \beta+77 \delta+31 \rho^{2}\right)-83 \lambda \rho\right)$ $+e^{2}\left(\alpha(223 \lambda+208 \rho \sigma)+\rho\left(\sigma\left(125 \beta+28 \rho^{2}\right)-10 \lambda \rho\right)+\delta(171 \lambda+232 \rho \sigma)\right)$

$\left.+e\left(14 \alpha^{2} \sigma+\alpha\left(\sigma\left(-27 \beta-28 \delta+172 \rho^{2}\right)+445 \lambda \rho\right)+27 \beta \delta \sigma+66 \beta \rho^{2} \sigma+168 \gamma \sigma+14 \delta^{2} \sigma+151 \delta \lambda \rho+78 \delta \rho^{2} \sigma+5 \lambda \rho^{3}+2 \rho^{4} \sigma\right)\right)$

$d_{7}=\frac{1}{2}\left(4 \alpha^{2} \sigma+2 \alpha\left(\sigma\left(23 \rho^{2}-4(\beta+\delta)\right)+68 \lambda \rho\right)+8 \beta \delta \sigma+9 \beta \rho^{2} \sigma+64 \gamma \sigma+4 \delta^{2} \sigma+36 \delta \lambda \rho+16 \delta \rho^{2} \sigma+2 e^{3}(16 \rho \sigma-19 \lambda)\right.$ $\left.+e^{2}\left(2 \sigma\left(32 \alpha+19 \beta+57 \delta+20 \rho^{2}\right)-15 \lambda \rho\right)+e\left(2 \alpha(76 \lambda+63 \rho \sigma)+\rho\left(\sigma\left(49 \beta+8 \rho^{2}\right)+9 \lambda \rho\right)+2 \delta(46 \lambda+53 \rho \sigma)\right)\right)$

$d_{8}=\frac{5}{2}(\alpha(8 \lambda+6 \rho \sigma)+\beta \rho \sigma+4 \delta(\lambda+\rho \sigma))+e^{2}(9 \rho \sigma-5 \lambda)+\frac{1}{2} e\left(2 \sigma\left(18 \alpha+5 \beta+23 \delta+5 \rho^{2}\right)+5 \lambda \rho\right)$

$d_{9}=2 \sigma(2(\alpha+\delta)+e \rho)$ 
Open Access. This article is distributed under the terms of the Creative Commons Attribution License (CC-BY 4.0), which permits any use, distribution and reproduction in any medium, provided the original author(s) and source are credited.

\section{References}

[1] J.M. Maldacena, The Large $N$ limit of superconformal field theories and supergravity, Int. J. Theor. Phys. 38 (1999) 1113 [hep-th/9711200] [InSPIRE].

[2] E. Witten, Anti-de Sitter space and holography, Adv. Theor. Math. Phys. 2 (1998) 253 [hep-th/9802150] [INSPIRE].

[3] J. Penedones, TASI lectures on AdS/CFT, in Proceedings, Theoretical Advanced Study Institute in Elementary Particle Physics: New Frontiers in Fields and Strings (TASI 2015), Boulder, CO, U.S.A., 1-26 June 2015, pp. 75-136 (2017) [DOI:10.1142/9789813149441_0002] [arXiv: 1608.04948] [INSPIRE].

[4] D. Poland, S. Rychkov and A. Vichi, The Conformal Bootstrap: Theory, Numerical Techniques and Applications, Rev. Mod. Phys. 91 (2019) 015002 [arXiv:1805.04405] [INSPIRE].

[5] R. Haag, Quantum field theories with composite particles and asymptotic conditions, Phys. Rev. 112 (1958) 669 [INSPIRE].

[6] R. Haag, The framework of quantum field theory, Nuovo Cim. 14 (1959) 131 [InSPIRE].

[7] D. Ruelle, On the Asymptotic Condition in Quantum Field Theory, Verlag nicht ermittelbar (1962).

[8] H. Hannesdottir and M.D. Schwartz, A Finite S-matrix, arXiv:1906.03271 [INSPIRE].

[9] J. Penedones, Writing CFT correlation functions as AdS scattering amplitudes, JHEP 03 (2011) 025 [arXiv: 1011.1485] [INSPIRE].

[10] D.Z. Freedman, S.D. Mathur, A. Matusis and L. Rastelli, Comments on 4 point functions in the CFT/AdS correspondence, Phys. Lett. B 452 (1999) 61 [hep-th/9808006] [INSPIRE].

[11] H. Liu and A.A. Tseytlin, On four point functions in the CFT/AdS correspondence, Phys. Rev. D 59 (1999) 086002 [hep-th/9807097] [INSPIRE].

[12] D.Z. Freedman, S.D. Mathur, A. Matusis and L. Rastelli, Correlation functions in the $C F T_{d} / A d S_{d+1}$ correspondence, Nucl. Phys. B 546 (1999) 96 [hep-th/9804058] [InSPIRE].

[13] E. D'Hoker, D.Z. Freedman and L. Rastelli, AdS/CFT four point functions: How to succeed at $z$ integrals without really trying, Nucl. Phys. B 562 (1999) 395 [hep-th/9905049] [INSPIRE].

[14] E. D'Hoker, D.Z. Freedman, S.D. Mathur, A. Matusis and L. Rastelli, Graviton exchange and complete four point functions in the AdS/CFT correspondence, Nucl. Phys. B 562 (1999) 353 [hep-th/9903196] [INSPIRE].

[15] R. Britto, F. Cachazo, B. Feng and E. Witten, Direct proof of tree-level recursion relation in Yang-Mills theory, Phys. Rev. Lett. 94 (2005) 181602 [hep-th/0501052] [INSPIRE].

[16] E. Witten, Perturbative gauge theory as a string theory in twistor space, Commun. Math. Phys. 252 (2004) 189 [hep-th/0312171] [INSPIRE]. 
[17] N. Arkani-Hamed, F. Cachazo and J. Kaplan, What is the Simplest Quantum Field Theory?, JHEP 09 (2010) 016 [arXiv:0808.1446] [INSPIRE].

[18] N. Arkani-Hamed, F. Cachazo, C. Cheung and J. Kaplan, A Duality For The S Matrix, JHEP 03 (2010) 020 [arXiv:0907.5418] [inSPIRE].

[19] N. Arkani-Hamed, F. Cachazo and C. Cheung, The Grassmannian Origin Of Dual Superconformal Invariance, JHEP 03 (2010) 036 [arXiv: 0909. 0483] [INSPIRE].

[20] M.F. Paulos, Towards Feynman rules for Mellin amplitudes, JHEP 10 (2011) 074 [arXiv:1107.1504] [INSPIRE].

[21] G. Mack, D-dimensional Conformal Field Theories with anomalous dimensions as Dual Resonance Models, Bulg. J. Phys. 36 (2009) 214 [arXiv:0909.1024] [inSPIRE].

[22] A.L. Fitzpatrick, J. Kaplan, J. Penedones, S. Raju and B.C. van Rees, A Natural Language for AdS/CFT Correlators, JHEP 11 (2011) 095 [arXiv: 1107.1499] [INSPIRE].

[23] S. Kharel and G. Siopsis, Tree-level Correlators of scalar and vector fields in AdS/CFT, JHEP 11 (2013) 159 [arXiv: 1308.2515] [INSPIRE].

[24] A.L. Fitzpatrick and J. Kaplan, Analyticity and the Holographic S-matrix, JHEP 10 (2012) 127 [arXiv: 1111.6972] [INSPIRE].

[25] A.L. Fitzpatrick and J. Kaplan, Unitarity and the Holographic S-matrix, JHEP 10 (2012) 032 [arXiv:1112.4845] [INSPIRE].

[26] M.S. Costa, V. Gonçalves and J. Penedones, Spinning AdS Propagators, JHEP 09 (2014) 064 [arXiv: 1404.5625] [INSPIRE].

[27] C.B. Jepsen and S. Parikh, Recursion Relations in p-adic Mellin Space, J. Phys. A 52 (2019) 285401 [arXiv: 1812.09801] [INSPIRE].

[28] C.B. Jepsen and S. Parikh, p-adic Mellin Amplitudes, JHEP 04 (2019) 101 [arXiv: 1808.08333] [INSPIRE].

[29] S.S. Gubser, C. Jepsen and B. Trundy, Spin in p-adic AdS/CFT, J. Phys. A 52 (2019) 144004 [arXiv: 1811.02538] [INSPIRE].

[30] E. Hijano, P. Kraus, E. Perlmutter and R. Snively, Witten Diagrams Revisited: The AdS Geometry of Conformal Blocks, JHEP 01 (2016) 146 [arXiv:1508.00501] [INSPIRE].

[31] E.Y. Yuan, Simplicity in AdS Perturbative Dynamics, arXiv:1801.07283 [INSPIRE].

[32] S. Parikh, Holographic dual of the five-point conformal block, JHEP 05 (2019) 051 [arXiv: 1901.01267] [INSPIRE].

[33] C.B. Jepsen and S. Parikh, Propagator identities, holographic conformal blocks and higher-point AdS diagrams, JHEP 10 (2019) 268 [arXiv: 1906.08405] [INSPIRE].

[34] S. Raju, New Recursion Relations and a Flat Space Limit for AdS/CFT Correlators, Phys. Rev. D 85 (2012) 126009 [arXiv:1201.6449] [INSPIRE].

[35] S. Raju, Four Point Functions of the Stress Tensor and Conserved Currents in $A d S_{4} / \mathrm{CFT}_{3}$, Phys. Rev. D 85 (2012) 126008 [arXiv: 1201.6452] [INSPIRE].

[36] J.M. Maldacena and G.L. Pimentel, On graviton non-Gaussianities during inflation, JHEP 09 (2011) 045 [arXiv: 1104.2846] [INSPIRE].

[37] N. Arkani-Hamed and J. Maldacena, Cosmological Collider Physics, arXiv:1503.08043 [INSPIRE]. 
[38] S. Albayrak, C. Chowdhury and S. Kharel, New relation for Witten diagrams, JHEP 10 (2019) 274 [arXiv : 1904.10043] [INSPIRE].

[39] S. Raju, BCFW for Witten Diagrams, Phys. Rev. Lett. 106 (2011) 091601 [arXiv: 1011.0780] [INSPIRE].

[40] I. Mata, S. Raju and S. Trivedi, CMB from CFT, JHEP 07 (2013) 015 [arXiv:1211. 5482] [INSPIRE].

[41] S. Raju, Recursion Relations for AdS/CFT Correlators, Phys. Rev. D 83 (2011) 126002 [arXiv: 1102.4724] [INSPIRE].

[42] A. Bzowski, P. McFadden and K. Skenderis, Implications of conformal invariance in momentum space, JHEP 03 (2014) 111 [arXiv:1304.7760] [INSPIRE].

[43] A. Bzowski, P. McFadden and K. Skenderis, Scalar 3-point functions in CFT: renormalisation, $\beta$-functions and anomalies, JHEP 03 (2016) 066 [arXiv:1510.08442] [INSPIRE].

[44] A. Bzowski, P. McFadden and K. Skenderis, Renormalised CFT 3-point functions of scalars, currents and stress tensors, JHEP 11 (2018) 159 [arXiv: 1805.12100] [INSPIRE].

[45] A. Bzowski, P. McFadden and K. Skenderis, Evaluation of conformal integrals, JHEP 02 (2016) 068 [arXiv: 1511.02357] [INSPIRE].

[46] J.M. Maldacena, Non-Gaussian features of primordial fluctuations in single field inflationary models, JHEP 05 (2003) 013 [astro-ph/0210603] [INSPIRE].

[47] H. Isono, T. Noumi and T. Takeuchi, Momentum space conformal three-point functions of conserved currents and a general spinning operator, JHEP 05 (2019) 057 [arXiv: 1903.01110] [INSPIRE].

[48] H. Isono, T. Noumi and G. Shiu, Momentum space approach to crossing symmetric CFT correlators, JHEP 07 (2018) 136 [arXiv:1805.11107] [INSPIRE].

[49] C. Corianò and M.M. Maglio, Exact Correlators from Conformal Ward Identities in Momentum Space and the Perturbative TJJ Vertex, Nucl. Phys. B 938 (2019) 440 [arXiv: 1802.07675] [INSPIRE].

[50] C. Corianò, M.M. Maglio, A. Tatullo and D. Theofilopoulos, Exact Correlators from Conformal Ward Identities in Momentum Space and Perturbative Realizations, PoS (CORFU2018) 072 (2019) [arXiv: 1904.13174] [INSPIRE].

[51] C. Corianò and M.M. Maglio, On Some Hypergeometric Solutions of the Conformal Ward Identities of Scalar 4-point Functions in Momentum Space, JHEP 09 (2019) 107 [arXiv: 1903.05047] [INSPIRE].

[52] M. Gillioz, Momentum-space conformal blocks on the light cone, JHEP 10 (2018) 125 [arXiv: 1807.07003] [INSPIRE].

[53] N. Arkani-Hamed, D. Baumann, H. Lee and G.L. Pimentel, The Cosmological Bootstrap: Inflationary Correlators from Symmetries and Singularities, arXiv:1811.00024 [INSPIRE].

[54] C. Corianò and M.M. Maglio, The general 3-graviton vertex (TTT) of conformal field theories in momentum space in $d=4$, Nucl. Phys. B 937 (2018) 56 [arXiv:1808.10221] [inSPIRE].

[55] C. Corianò, L. Delle Rose, E. Mottola and M. Serino, Solving the Conformal Constraints for Scalar Operators in Momentum Space and the Evaluation of Feynman's Master Integrals, JHEP 07 (2013) 011 [arXiv:1304.6944] [InSPIRE]. 
[56] S. Albayrak and S. Kharel, Towards the higher point holographic momentum space amplitudes, JHEP 02 (2019) 040 [arXiv:1810.12459] [INSPIRE].

[57] A.H. Guth, The Inflationary Universe: A Possible Solution to the Horizon and Flatness Problems, Phys. Rev. D 23 (1981) 347 [inSPIRE].

[58] A.D. Linde, A New Inflationary Universe Scenario: A Possible Solution of the Horizon, Flatness, Homogeneity, Isotropy and Primordial Monopole Problems, Phys. Lett. 108B (1982) 389 [INSPIRE].

[59] A. Albrecht and P.J. Steinhardt, Cosmology for Grand Unified Theories with Radiatively Induced Symmetry Breaking, Phys. Rev. Lett. 48 (1982) 1220 [INSPIRE].

[60] A.A. Starobinsky, Dynamics of Phase Transition in the New Inflationary Universe Scenario and Generation of Perturbations, Phys. Lett. 117B (1982) 175 [INSPIRE].

[61] P. McFadden and K. Skenderis, Holography for Cosmology, Phys. Rev. D 81 (2010) 021301 [arXiv:0907.5542] [INSPIRE].

[62] X. Chen and Y. Wang, Quasi-Single Field Inflation and Non-Gaussianities, JCAP 04 (2010) 027 [arXiv: 0911.3380] [INSPIRE].

[63] D. Baumann and D. Green, Signatures of Supersymmetry from the Early Universe, Phys. Rev. D 85 (2012) 103520 [arXiv:1109.0292] [INSPIRE].

[64] V. Assassi, D. Baumann and D. Green, On Soft Limits of Inflationary Correlation Functions, JCAP 11 (2012) 047 [arXiv:1204.4207] [InSPIRE].

[65] X. Chen and Y. Wang, Quasi-Single Field Inflation with Large Mass, JCAP 09 (2012) 021 [arXiv: 1205.0160] [INSPIRE].

[66] V. Assassi, D. Baumann, D. Green and L. McAllister, Planck-Suppressed Operators, JCAP 01 (2014) 033 [arXiv:1304.5226] [INSPIRE].

[67] H. Lee, D. Baumann and G.L. Pimentel, Non-Gaussianity as a Particle Detector, JHEP 12 (2016) 040 [arXiv: 1607.03735] [INSPIRE].

[68] H. An, M. McAneny, A.K. Ridgway and M.B. Wise, Quasi Single Field Inflation in the non-perturbative regime, JHEP 06 (2018) 105 [arXiv: 1706.09971] [INSPIRE].

[69] A. Kehagias and A. Riotto, On the Inflationary Perturbations of Massive Higher-Spin Fields, JCAP 07 (2017) 046 [arXiv: 1705.05834] [INSPIRE].

[70] S. Kumar and R. Sundrum, Heavy-Lifting of Gauge Theories By Cosmic Inflation, JHEP 05 (2018) 011 [arXiv: 1711.03988] [INSPIRE].

[71] D. Baumann, G. Goon, H. Lee and G.L. Pimentel, Partially Massless Fields During Inflation, JHEP 04 (2018) 140 [arXiv:1712.06624] [INSPIRE].

[72] G. Franciolini, A. Kehagias and A. Riotto, Imprints of Spinning Particles on Primordial Cosmological Perturbations, JCAP 02 (2018) 023 [arXiv:1712.06626] [INSPIRE].

[73] G. Goon, K. Hinterbichler, A. Joyce and M. Trodden, Shapes of gravity: Tensor non-Gaussianity and massive spin-2 fields, JHEP 10 (2019) 182 [arXiv:1812.07571] [INSPIRE].

[74] D. Anninos, V. De Luca, G. Franciolini, A. Kehagias and A. Riotto, Cosmological Shapes of Higher-Spin Gravity, JCAP 04 (2019) 045 [arXiv: 1902.01251] [INSPIRE]. 
[75] S. Pi and M. Sasaki, Curvature Perturbation Spectrum in Two-field Inflation with a Turning Trajectory, JCAP 10 (2012) 051 [arXiv: 1205.0161] [INSPIRE].

[76] J.-O. Gong, S. Pi and M. Sasaki, Equilateral non-Gaussianity from heavy fields, JCAP 11 (2013) 043 [arXiv: 1306.3691] [INSPIRE].

[77] A. Bzowski, P. McFadden and K. Skenderis, Renormalised 3-point functions of stress tensors and conserved currents in CFT, JHEP 11 (2018) 153 [arXiv:1711.09105] [INSPIRE].

[78] S.Y. Li, Y. Wang and S. Zhou, KLT-Like Behaviour of Inflationary Graviton Correlators, JCAP 12 (2018) 023 [arXiv: 1806.06242] [INSPIRE].

[79] J.A. Farrow, A.E. Lipstein and P. McFadden, Double copy structure of CFT correlators, JHEP 02 (2019) 130 [arXiv: 1812.11129] [INSPIRE].

[80] C. Sleight, A Mellin Space Approach to Cosmological Correlators, arXiv:1906.12302 [INSPIRE].

[81] C. Sleight and M. Taronna, Bootstrapping Inflationary Correlators in Mellin Space, arXiv: 1907.01143 [INSPIRE].

[82] P. Benincasa, From the flat-space S-matrix to the Wavefunction of the Universe, arXiv: 1811.02515 [INSPIRE].

[83] N. Arkani-Hamed and P. Benincasa, On the Emergence of Lorentz Invariance and Unitarity from the Scattering Facet of Cosmological Polytopes, arXiv:1811.01125 [INSPIRE].

[84] N. Arkani-Hamed, P. Benincasa and A. Postnikov, Cosmological Polytopes and the Wavefunction of the Universe, arXiv:1709.02813 [INSPIRE]. 